\title{
Review Article \\ Recent Advances in Unconventional Lithography for Challenging 3D Hierarchical Structures and Their Applications
}

\author{
Jong Uk Kim, ${ }^{1,2}$ Sori Lee, ${ }^{2}$ and Tae-il Kim ${ }^{1,2}$ \\ ${ }^{1}$ Center for Neuroscience Imaging Research, Institute of Basic Science (IBS), Suwon 440-746, Republic of Korea \\ ${ }^{2}$ School of Chemical Engineering, Sungkyunkwan University (SKKU), Suwon 440-746, Republic of Korea \\ Correspondence should be addressed to Tae-il Kim; taeilkim@skku.edu
}

Received 19 October 2015; Accepted 7 February 2016

Academic Editor: Bo Tan

Copyright (c) 2016 Jong Uk Kim et al. This is an open access article distributed under the Creative Commons Attribution License, which permits unrestricted use, distribution, and reproduction in any medium, provided the original work is properly cited.

\begin{abstract}
In nanoscience and nanotechnology, nanofabrication is critical. Among the required processes for nanofabrication, lithography is one of core issues. Although conventional photolithography with recent remarkable improvement has contributed to the industry during the past few decades, fabrication of 3-dimensional (3D) nanostructure is still challenging. In this review, we summarize recent advances for the construction of 3D nanostructures by unconventional lithography and the combination of two top-down approaches or top-down and bottom-up approaches. We believe that the 3D hierarchical nanostructures described here will have a broad range of applications having adaptable levels of functional integration of precisely controlled nanoarchitectures that are required by not only academia, but also industry.
\end{abstract}

\section{Introduction}

Nanofabrication is a "hot topic" in nanotechnology and nanoscience. It is absolutely indispensable to solve all scientific questions in nanoscale photonics, biotechnology, and electronics. However, with every increasing demand for smaller features on integrated and complicated geometries, its realization in industry relies on photo (or electron-beam) lithography which has limitations based on the physics on light diffraction and poor throughput that are impractical to overcome [1]. Conventional techniques are reaching their resolution limits and have relatively high cost for the fabrication of patterned nanostructures. On the other hand, unconventional lithography techniques were first seen in early 1990's [2-6]. Interestingly, at that time, new research had been exploring nanoimprint lithography (NIL) [7], capillary force lithography (CFL) [8, 9], dip-pen lithography [10], soft molding [11, 12], microcontact printing $(\mu \mathrm{CP})[2,13]$, and various types of self-assembly $[14,15]$. They provided various alternative approaches and unique accomplishments: diverse, novel, and reliable processes to reliably generate smaller pattern features (sub-100 nm scale) with high cost effectiveness. Because there are significant potential and substantial developments for academia and small companies, unconventional lithography has been explosively developed. Therefore, the number of scientific publications and citations of unconventional lithography has been dramatically increasing in the last 20 years [16]. Even with the recent remarkable improvements, unconventional lithography still has huge hurdles, for example, multiple aspect-ratio (3D hierarchical) nanostructures, large area, and low-cost manufacturing with sub-10 $\mathrm{nm}$ feature sizes [17-19]. In this review article, we summarize previously demonstrated unconventional lithography and introduce the challenging fields of 3D hierarchical nanostructures and their applications. The paper is composed of five separate parts. The first part presents unconventional lithography methods developed in the last 20 years. The second and third parts deal with 3D hierarchical nanostructures and their applications formed by molding or photolithography (combined top-down lithographic techniques). The fourth and fifth parts cover integrated assembly with merged top-down with bottom-up approaches.

\section{Unconventional Lithography}

In this section, we attempt to summarize representative sampling of unconventional lithography (Figure 1). The processes 


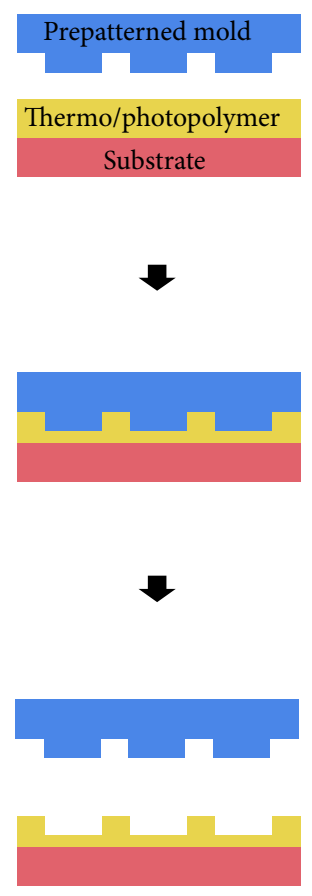

(a)
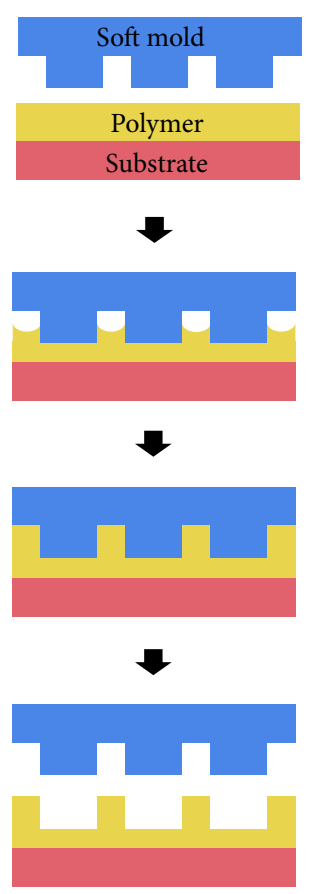

(b)

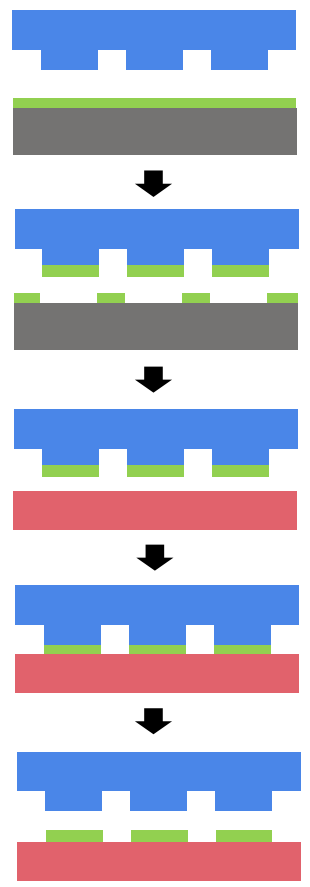

(c)

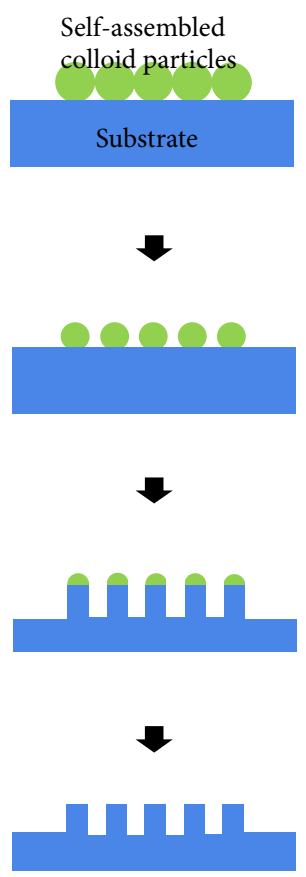

(d)

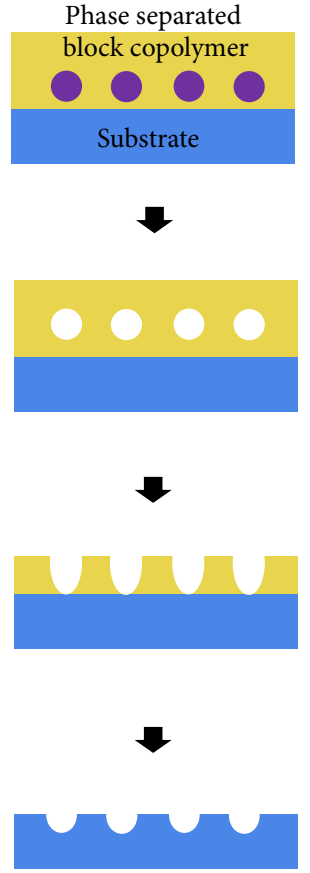

(e)

FIGURE 1: Various unconventional lithographic processes. Schematic illustration of (a) thermal nanoimprint lithography (T-NIL, also called hot embossing) and UV nanoimprint lithography (UV-NIL, also called photocurable nanoimprint lithography), (b) capillary force lithography (CFL), (c) nanotransfer printing lithography (NTL), (d) colloidal lithography (CL, also called nanosphere lithography), and (e) block copolymer lithography (BCPL).

of making functional structures with patterns are labeled as top-down (NIL, Figure 1(a); CFL, Figure 1(b); nanotransfer printing (NTL), Figure 1(c)) and bottom-up (colloidal lithography (CL), Figure 1(d), and block copolymer (BCP) lithography, Figure 1(e)) approaches.

\subsection{Unconventional top-down Lithography}

2.1.1. Nanoimprint Lithography (NIL). The process of NIL was first demonstrated by Chou et al. in 1995 [7]. The concept of the technique is mechanical replication where surface reliefs from prepatterned mold are embossed into a thin layer on the substrate [20,21]. Figure 1(a) gives an overview of the NIL processing steps. In the NIL, resist layer (with thermo- $[22,23]$ or photocurable polymer [24]) is coated on a substrate and then pressed by a rigid mold with patterns at $1: 1$ scale through mechanical contact. The resist layer is cured with pressure by thermal or UV curing known, respectively, as temperature-based processing and light-initiated polymerization. In principle, NIL does not have limitations of pattern geometry, which means NIL can copy any patterns on a wide range of substrates. Therefore, NIL can bridge the gap between lab level nanobased technique studies and production level manufacturing. However, it requires high pressure $(\sim \mathrm{MPa})$ and new challenging processes for large area fabrications, that is, roll-to-roll [25-27] or step-and-repeat processes [28].
2.1.2. Capillary Force Lithography (CFL). CFL is a method that uses nature force, capillarity with soft or flexible nanoscale molds. Lee and Suh $[8,9]$ presented large-area patterning by capillarity of melted polymer or polymeric precursors. In this method, by using patterned soft mold such as polydimethylsiloxane (PDMS) or rigiflex molds, thin polymer layers on the substrate are contacted and then heated above the polymer's glass transition temperature $\left(T_{g}\right)$. Laplace pressure induced by a micro/nanoscale mold allows the melted polymer to fill the void space of the channels formed between the mold and the polymer without applying pressure (Figure 1(b)) [29,30]. Notably, the strength of CFL is that it allows for smaller sizes of feature patterns with higher Laplace pressure and higher throughput [31]. However, CFL is still limited in forming high aspect and multiple geometry nanostructures.

2.1.3. Nanotransfer Printing (NTP). Another method to achieve aligned nanopatterns over a large area is to transfer the desired pattern to the target substrate [32-40]. This technique, known as NTP, was introduced by Rogers et al. [3234]. As shown in Figure 1(c), patterned structures deposited on a polymeric stamp are selectively transferred onto different surfaces. First a stamp contacts a donor substrate coated with the target structure and then quickly peels it away. After the micro/nanostructures are transferred from the donor substrate to the stamp, the stamp contacts the receiving substrate 
and then slowly peels it away. Consequently the structures are transferred from the stamp to the receiver. In previous studies, ultrathin inorganic devices like single crystal silicon nanoribbons [41-43], photodiodes [44-47], and solar cells were printed onto a sheet of plastic by kinetically adhesioncontrollable stamp [34]. These results show that NTP has potential to be useful to transfer conventional electronics to deformable substrates for flexible and stretchable devices. Also, NTP might be used for many other applications in biomedicine, that is, optogenetics [48, 49], catheter [50], and optoelectronics. Although recent advances as mentioned above are presented, it is still suffering from low throughout and poor yield for large area fabrication.

\subsection{Unconventional Bottom-Up Lithography}

2.2.1. Colloidal Lithography (CL). Colloidal lithography (CL) uses an array of self-organized and self-assembled colloids as templates for advanced functional materials [51-55]. CL has simple steps to create nanostructures [51]; a close packed monolayer of colloids (nanosphere) arranged into an array through self-assembly on a substrate. And then reactive ion etching (RIE), such as plasma etching, fabricates nanopillar structures (Figure 1(d)). A remarkable characteristic of the process is that the colloids assemble themselves spontaneously in ordered architectures which are easily controlled over a large area without complex equipment. Modification of the self-assembled etch mask improves the versatility of CL in fabricating nanopatterns such as hollow shells [52], nanocylinders [53], and even multiple layer $[54,55]$ by spontaneous formation of well-ordered colloid. Therefore this fabrication method has some advantages over conventional lithography; it is a simple cost-effective process that provides easy control of feature size.

\subsubsection{Block Copolymer Lithography. Another self-assembled} material is block copolymers (BCPs). BCPs are macromolecules consisting of more than two components with chemically different polymeric segments [56-58]. Owing to their segmented structures, BCPs have various shapes of phase separation with different processing conditions with well-aligned arrays at equilibrium with lots of complex morphologies, from lamellar to cylinder (or gyroid) nanoscale structures (Figure 1(e)) [15]. Conveniently, BCP lithography is compatible with conventional manufacturing processes used in the semiconductor industry, showing a wide range of potential as an emerging technology for nanoscale device fabrication.

\section{Unconventional Lithography for 3D Hierarchical Micro- and Nanostructure by Combined Top-Down Approaches}

All approaches we mentioned in Section 2 demonstrate only monoscale micro- or nanostructures with single step process. Although the previous approaches are useful in many fields, more complicated, denser, and 3-dimensional hierarchical patterns with higher aspect ratio are also required. In this section, we describe several approaches to fabricate high aspect ratio hierarchical structures having nanoscale patterns on microscale structures by using combined top-down lithographic techniques (Figure 2). Precisely controlled hierarchical patterning at multilevel and size-control of individual structures is possible by the techniques. As a result, the range of its applications is being widened.

3.1. Two-Step Photolithography for 3D Hierarchical Structure. Greiner et al. [59] reported production of multiscale micropillar structured PDMS using two-step photolithography. The process to fabricate the hierarchical structure involves double layer-by-layer (LbL) coating and exposure steps using SU-8 resist (Figure 2(a)). A single development step after the second exposure was sufficient to remove the unexposed regions from the double layers. Subsequently, the 3D hierarchical PDMS replica was fabricated by soft molding on hierarchical SU-8 hole patterns. Fabricated PDMS replicas were characterized by scanning electron microscopy (SEM) (Figure 2(b)). The bottom pillars had a diameter of $50 \mu \mathrm{m}$ and a height of $200 \mu \mathrm{m}$ (aspect ratio $\lambda=4$ ), while the top pillars had a diameter of $5 \mu \mathrm{m}$ and heights ranging from 2.5 to $10 \mu \mathrm{m}$ (aspect ratio $\lambda$ from 0.2 to 2 ). However, in the aspect of practical application, this approach has lack of processibility due to highly viscous photoresist onto pre-patterned structures.

3.2. Sequential Thermal Nanoimprint Lithography ( $t$-NIL) Steps Assisted 3D Hierarchical Nanostructures. Although NIL has been recognized as a simple, low-cost, high-throughput, and scalable nanofabrication technique $[7,22,23]$, it is difficult to form high aspect ratio patterns with thermal NIL [60]. The large contact areas between the mold and melted polymer and limited mold materials that cannot be controlled in surface energy allow multiple $(\sim 2)$ aspect ratio patterns to be possible. Recently, Zhang and Low [61] demonstrated a new fabrication method for 3D hierarchical structures by NIL by exploiting the properties of thermoplastic polymers. The sequential step process for fabrication of the $3 \mathrm{D}$ hierarchical structure is shown in Figure 2(c). First, a mold is pressed on a polymeric film above its glass transition temperature $T_{g}$. After demolding, a different mold is aligned with a specific orientation and pressed on the prepatterned polymer film below its glass transition temperature $T_{g}$ in order to fabricate a secondary pattern. In the same manner, a tertiary or higherorder pattern can also be created. Figure 2(d) shows scanning electron microscopy (SEM) images for the imprint of $2 \mu \mathrm{m}$ and $250 \mathrm{~nm}$ Si grating molds (top images). SEM images show polycarbonate (PC) film imprinted first with $2 \mu \mathrm{m}$ grating and then with $250 \mathrm{~nm}$ grating in parallel orientation and tertiary $250 \mathrm{~nm}$ grating imprinted perpendicular to other gratings (bottom images). They demonstrated that this approach offers a fast low-cost process to fabricate $3 \mathrm{D}$ hierarchical structures. However, the rigid and thick Si mold could lead to poor yield of demolding and it is difficult to control the temperature to prevent collapse or reflow of prepatterned structures [62]. Therefore, to extend this method, a highly precise temperature-controllable and rigiflex mold [39] could enhance the yield and reduce mechanical 


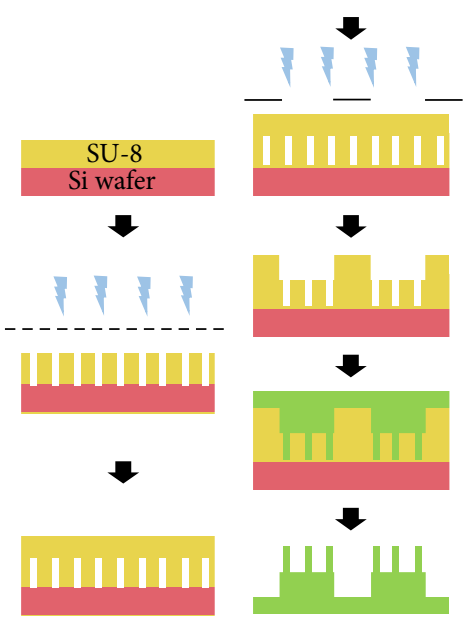

(a)
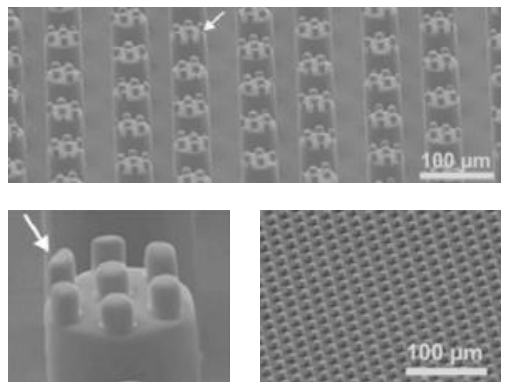

(b)
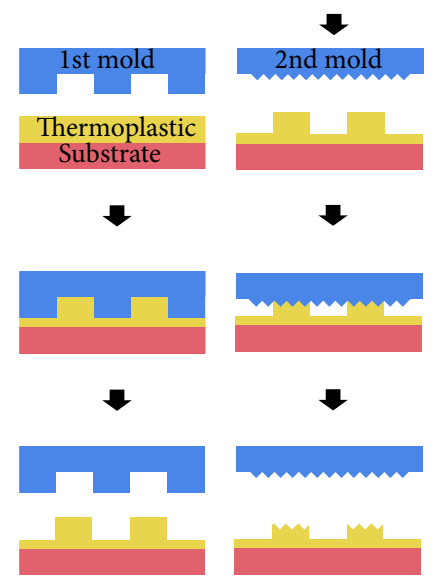

(c)
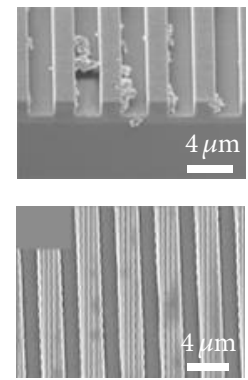

(d)
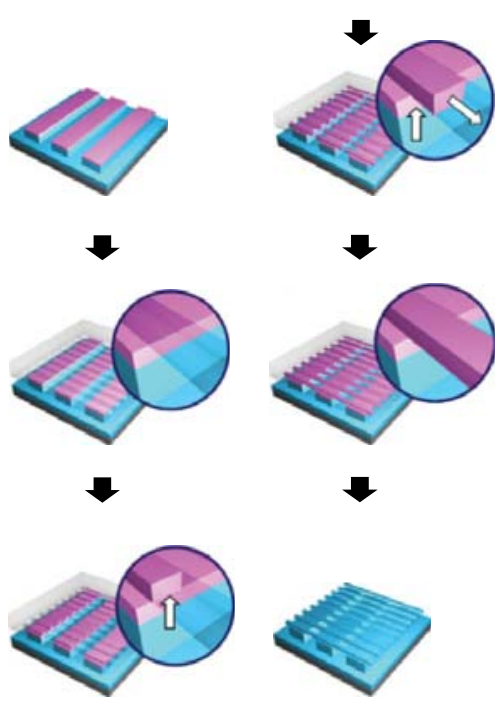

(e)
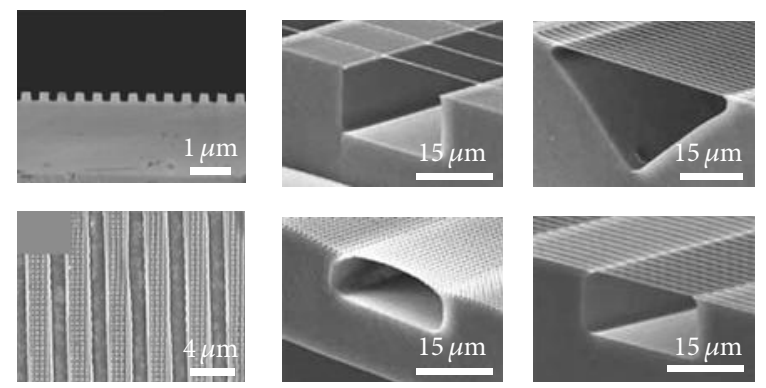

(f)

FIGURE 2: Schematic illustrations of fabrication process for 3D hierarchical micro- and nanostructures via (a) two-step photolithography and soft lithography, (c) sequential t-NIL, and (e) vacuum-assisted CFL. (b) SEM images showing the fabricated 3D micro/nano-hierarchical structures via two-step photolithography, followed by molding. Reproduced with permission from [59]. Copyright 2009 WILEY-VCH Verlag $\mathrm{GmbH} \& \mathrm{Co}$. KGaA. The bottom left SEM image shows close-up view of the selected area in top SEM image. Right SEM image of the bottom shows single-level pillars. (d) SEM images show the tilted cross section of the primary imprint $2 \mu \mathrm{m}$ (top left) grating mold and the secondary imprint $250 \mathrm{~nm}$ (top right) grating mold. The hierarchical structures were obtained from two steps (bottom left) and three steps (bottom right) of imprinting. Reproduced with permission from [61]. Copyright 2006 IOP Publishing Ltd. (f) SEM images show the several connected bridges of different width and density for given base structures via vacuum-assisted CFL. Reproduced with permission from [71]. Copyright 2009 Wiley-VCH Verlag GmbH \& Co. KGaA.

damage of both stamp and imprinted hierarchical structures as the alternatives to rigid Si molds.

\subsection{Vacuum-Assisted CFL for Fabricating 3D Hierarchi-} cal Bridge Structure. Hierarchically suspended polymericbridge structures have a wide range of applications as smart electronics, photonic devices, and microfluidic systems. Earlier available fabrication techniques including reverse imprint lithography [63, 64], microtransfer molding [65], edge lithography [66, 67], direct drawing [68], and electrochemical growth $[69,70]$ have been used to create bridge-like inverted $3 \mathrm{D}$ nanostructures. Although these approaches are useful, such suspended structures might contain a heterogeneous interface between their top and bottom structures, which is associated with structural defects and increases contact failure in electronic devices as well as partial current leakage in multichannel devices [71].
Recently, a novel approach to generate 3D hierarchical bridge structures was proposed by Kwak et al. in 2009 [71]. They combined partially curing UV-NIL and vacuumassisted CFL. As shown in Figure 2(e), vacuum-assisted CFL consists of three steps: (i) the first molding (the UV curable polymer surface is partially cured), (ii) the second molding, and (iii) vacuum-assisted molding. Fundamentally, oxygen inhibits UV-crosslinking since it reacts with scavenging initiator radicals in free-radical polymerization. These seemingly undesirable inhibitory effects form partially uncured regions of pregenerated microstructures. After fabricating a partially cured base microstructure (the first molding step), capillarity action of the partially cured resin simultaneously took place (the second molding step). Finally in the vacuumassisted molding step, the migration of a partially cured polymer was used to create $3 \mathrm{D}$ monolithic bridge like structures. The polymer then spontaneously moved into 
the second mold's cavity by capillary action and then fully cured after UV exposure. Figure 2(f) shows SEM images of various monolithic nanowire bridges of different width and density for a given base structure formed by the vacuumassisted CFL method. The method could serve as a novel tool for fabricating 3D hierarchical structures in a wide range of applications.

\section{Diverse Applications with 3D Hierarchical Structure}

In general, $3 \mathrm{D}$ hierarchical structure fabricated by combined top-down approaches leads to physically and mechanically unique properties. In this section, we introduce several applications using the 3D hierarchical structure and how to realize important properties including hydrophobicity, adhesion force, and robustness compared with conventional methods (Figure 3).

4.1. Directional Wetting and Spreading on Hierarchical Patterns. Anisotropic wetting and spreading of water droplet have attracted much attention [72-75] due to an ability to control liquid flow in a desired direction, which can be applied to microfluidic devices and a unidirectional wetting system, similar to the properties found on butterfly wings $[76,77]$, shark skin [78], and rice leaves [79]. Natural rice leaf possesses a hierarchical structure where the micropillars, with average diameter of about $5-8 \mu \mathrm{m}$, are arranged in parallel with nanoprotrusions on the top of the surface [80]. As a result, the surfaces of rice leaves present anisotropic wettability. Also, beetles that live in the Nambia desert have remarkable water collecting ability using their shell's nanoarchitectures that induce a gradient of hydrophobicity [81].

Recently, Radha et al. [82] applied sequential imprinting to mimic the hierarchical structures of rice leaves. They created a metallic rice leaf structure not the hierarchical patterning of the polymers. The advantage of this hierarchical metal surface allows it to survive in harsher environments, where polymers are infeasible, and to obtain highly desirable abilities, including anticorrosion, anti-icing, and selfcleaning $[82,83]$. The metallic patterns inspired by the rice leaf were generated by palladium $(\mathrm{Pd})$ benzylthiolate (metalorganic ink). The ink was first imprinted using a primary grating with a $2 \mu \mathrm{m}$ mold, and then fine pillars $(200 \mathrm{~nm}$ ) were patterned. Figure 3(a) shows SEM image of the rice leaf-like Pd structure with primary lines of $1.7 \mu \mathrm{m}$ and secondary pillars of $90 \mathrm{~nm}$ and corresponding height of $170 \mathrm{~nm}$ and $60 \mathrm{~nm}$, respectively, after heat treatment to remove organic materials. The structure has $130^{\circ}$ and $92^{\circ}$ contact angles, respectively, in parallel and perpendicular directions. To make a more hydrophobic surface, hexadecanethiol molecules were used on the metallic rice leaf surface (Figure 3(b)). In this case, this approach was a versatile method for fabricating the hierarchically hydrophobic metal surface.

4.2. Dry Adhesive Inspired by Gecko Using Hierarchical Micro/Nanostructures. Hierarchical structures can be used as bioinspired dry adhesives. Owing to an increase in the contact area by the high aspect ratio hierarchical structure, the adhesion induced by van der Waals force can be maintained even on a rough surface (roughness $<20 \mu \mathrm{m}$ ). These dry adhesives, for example, gecko-like structures, are useful for a wide range of applications including transportation devices [84], NTP [85], and wall climbing robots [86]. In particular, a dry adhesive patch with hierarchy has several advantages, including restoration of adhesion force, self-cleaning capability, and biocompatibility, in medical bandages, compared to commonly used bandages [8791].

Kwak et al. [90] and Bae et al. [91] reported interesting mushroom like micropillars. They were used with a commercially available unit of skin patch electrode which can monitor biosignals (Figures 3(c) and 3(d)). As shown in Figure 3(e), a widely used acrylic medical patch has several side effects such as redness, allergic response, and adhesion degradation by repeated attachment/detachment. On the other hand, the dry adhesive skin patch shows reduced pain during peel-off and undesirable side effects even after $48 \mathrm{~h}$ of use. Also, the dry adhesive patch has more restorable ability after every five cycles with the help of self-cleaning with water compared with widely used acrylic medical patches (Figure 3(f)). Hence, with increasing demands on long-term uses for the ubiquitous healthcare (U-healthcare) industry, hierarchically bioinspired dry adhesive patches will potentially be of great benefit in longitudinal biosignal acquisition.

\subsection{Free Standing Polymeric Membrane with Three-Level Hier-} archical Apertures. Free standing micro/nanomembranes have a wide range of applications including molecular separation [92-94], shadow masking [95], plasmonics [96-98], energy devices [99-101], and bioinspired microfluidic device $[102,103]$. Free standing silicon nitride $\left(\mathrm{SiN}_{x}\right)$ membranes are commonly used due to the high mechanical rigidity (Young's modulus, $E>130 \mathrm{GPa}$ ), which can withstand external forces during the handing process $[94,95,104-106]$. However, a $\mathrm{SiN}_{x}$ membrane is fragile under mechanical contact and requires expensive and complicated fabrication processes via e-beam lithography or focused ion-beam milling.

Cho et al. [107] created a new type of flexible, mechanically stable, and free standing polymeric membrane by using a hierarchical mold-based CFL. As shown in Figures 3(g) and $3(\mathrm{~h})$, the robust membrane with three-level hierarchical apertures $(800 \mu \mathrm{m} / 20 \mu \mathrm{m} / 500 \mathrm{~nm})$ was well defined without structure collapses or defects over a large area. Also, because the hierarchical membrane is easy to handle, it is easy to attach to a tip of the syringe to reorganize emulsions (Figure 3(i)). As a result, highly uniform and skewed distributions of emulsions depending on the aperture size were obtained; for example, $800 \mathrm{~nm}$ and $350 \mathrm{~nm}$ pores can distribute the mean sizes of $927.1 \mathrm{~nm}$ and $414.4 \mathrm{~nm}$, respectively (Figure $3(j)$ ). The results suggest that the mechanically stable membrane with hierarchical apertures is entirely possible to reorganize and generate highly uniform emulsions and is useful also in many fields. 

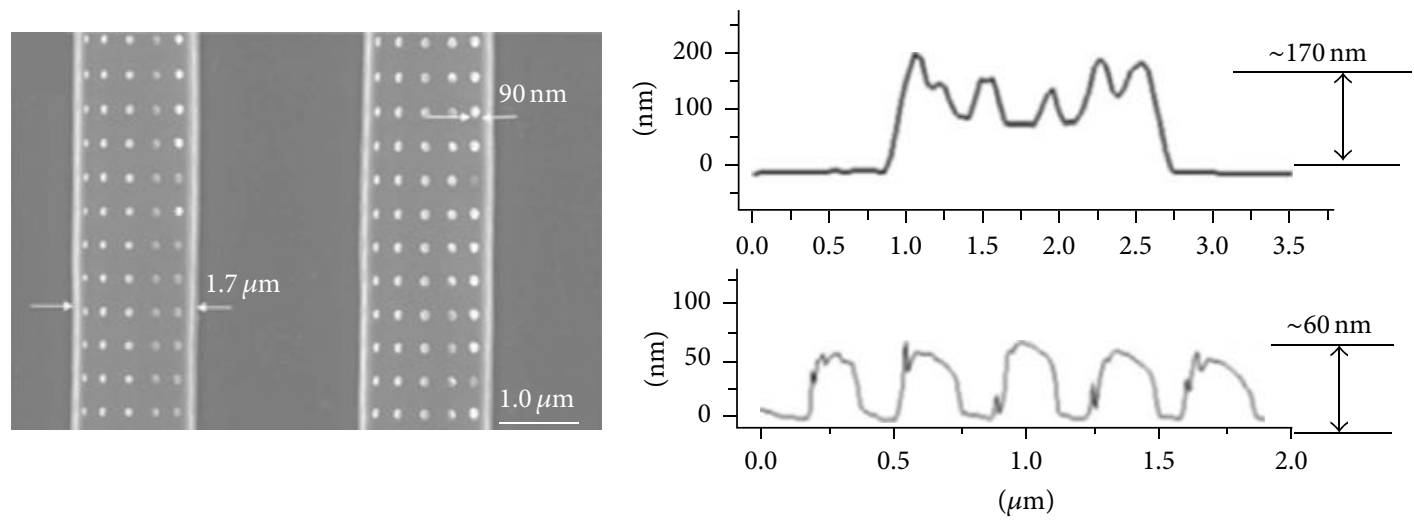

(a)
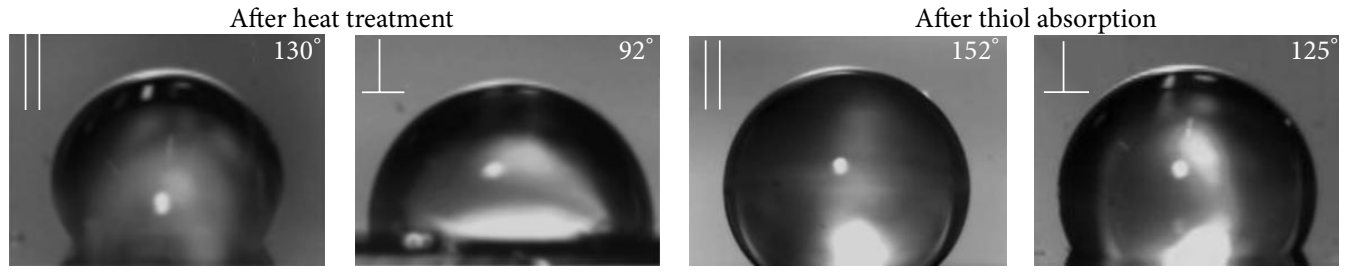

(b)

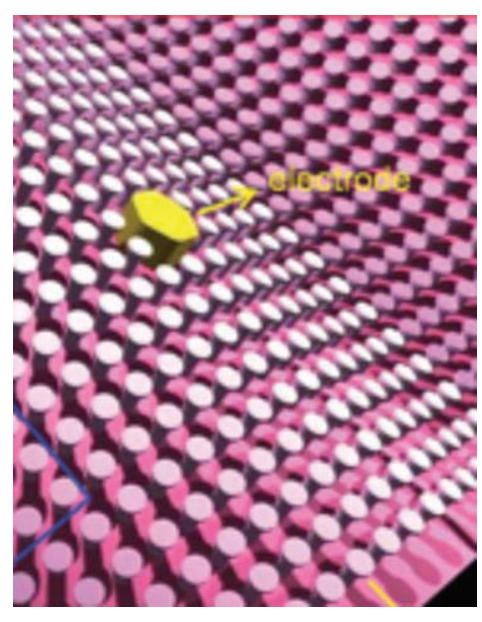

(c)
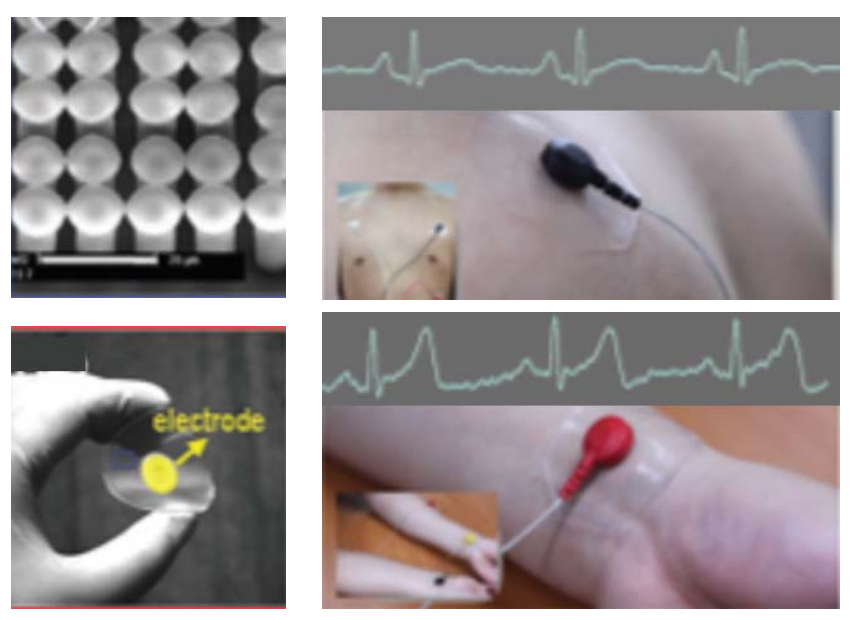

(d)

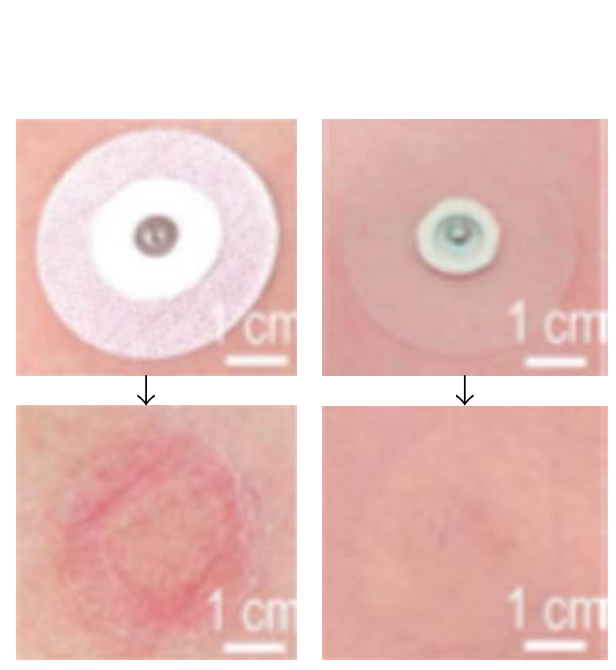

(e)

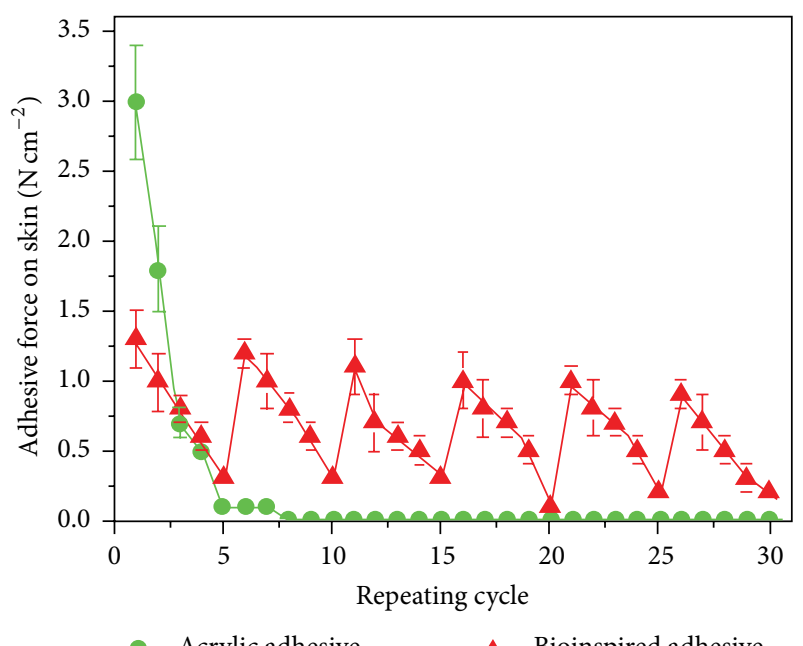

- Acrylic adhesive

$\neg$ Bioinspired adhesive

(f)

Figure 3: Continued. 


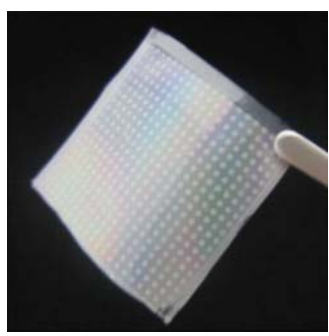

(g)

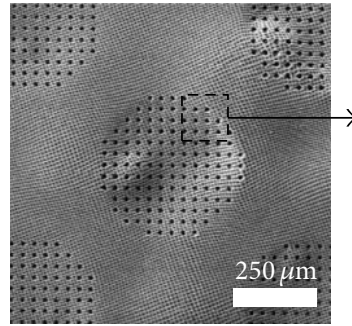

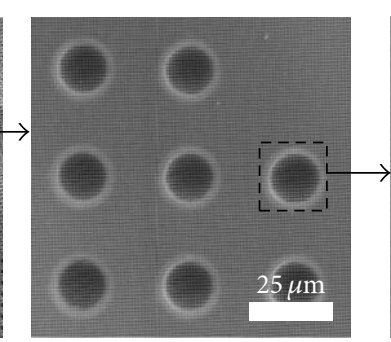

(h)

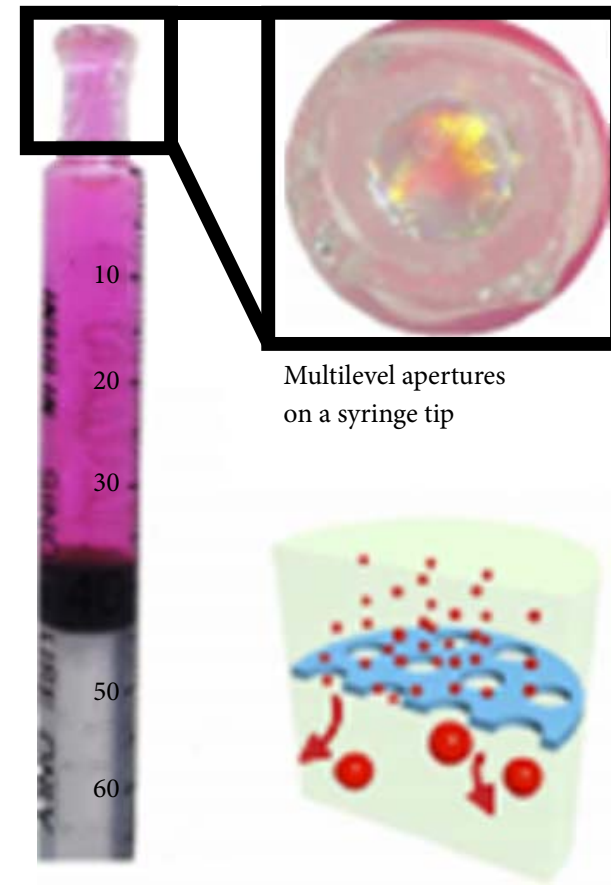

(i)

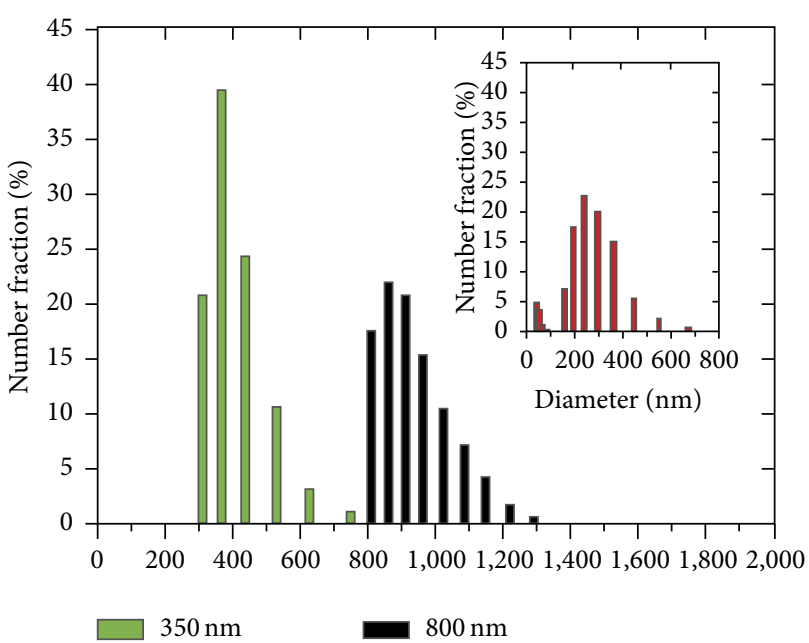

(j)

FIGURE 3: Applications of 3D hierarchical structures fabricated by combined top-down lithographical techniques. (a-b) (a) SEM image of the rice leaf-like hierarchical Pd structures (after heat treatment) with primary lines of $1.7 \mathrm{~mm}$ and secondary pillars of $90 \mathrm{~nm}$ and corresponding height of $170 \mathrm{~nm}$ and $60 \mathrm{~nm}$, respectively, from AFM profiles. (b) The different contact angles in parallel and perpendicular direction of the metallic rice leaf structure due to anisotropic wetting property (left). After hexadecanethiol absorption, the contact angle is increased in both directions (right). Reproduced with permission from [82]. Copyright 2013 Macmillan Publishers Ltd. (c-f) (c) Conceptual illustration (left), SEM image (top right), and photograph (bottom right) of bioinspired dry adhesive as medical skin patch. (d) Photographs show the use of dry adhesive patch to monitor electrocardiograms (ECGs) on the volunteer's chest (top) and wrist (bottom). The inset images show the corresponding ECG signals from the volunteer's heart. (e) Photographs show the skin condition after use of acrylic adhesive (left) and bioinspired dry adhesive during 48 hours. (f) Variations in normal adhesion strength of acrylic adhesive and bioinspired dry adhesive on the skin with 30 repeating cycles. Both adhesives were cleaned every 5 times. Reproduced with permission from [90, 91]. Copyright 2011 and 2013 WILEY-VCH Verlag GmbH \& Co. KGaA. (g-j) Large-area photograph (g) and SEM images (h) of the three-level hierarchical apertures $(800 \mathrm{~nm} / 20 \mu \mathrm{m} / 500 \mu \mathrm{m}$ apertures) at different magnifications. (i) Photographs of the three-level polymeric membrane with hierarchical apertures onto a tip of a syringe. The inset illustration depicts the reorganization process near the apertures. (j) Size distributions of emulsions before and after the filtration of apertures having two different diameters ( $800 \mathrm{~nm}$ apertures and $350 \mathrm{~nm}$ apertures). Reproduced with permission from [107]. Copyright 2014 Macmillan Publishers Ltd.

\section{Integrating Assembly: Merging Top- Down and Bottom-Up Approach in Unconventional Lithography}

Despite extensive efforts to develop nanofabrication, a superior technique enabling sub-10 nm scale control as well as large-area patterning is still required. Here, change toward integrated top-down and bottom-up lithographic technique might be a breakthrough strategy. In this section, we present some approaches for integrating assembly combined topdown and bottom-up unconventional lithography (Figure 4).

5.1. Template-Assisted Self-Assembly of Spherical Colloids. An interesting approach to control assembly of spherical colloids was proposed by Xia et al. [108, 109]. This method is based on combining physical templating and self-assembly 


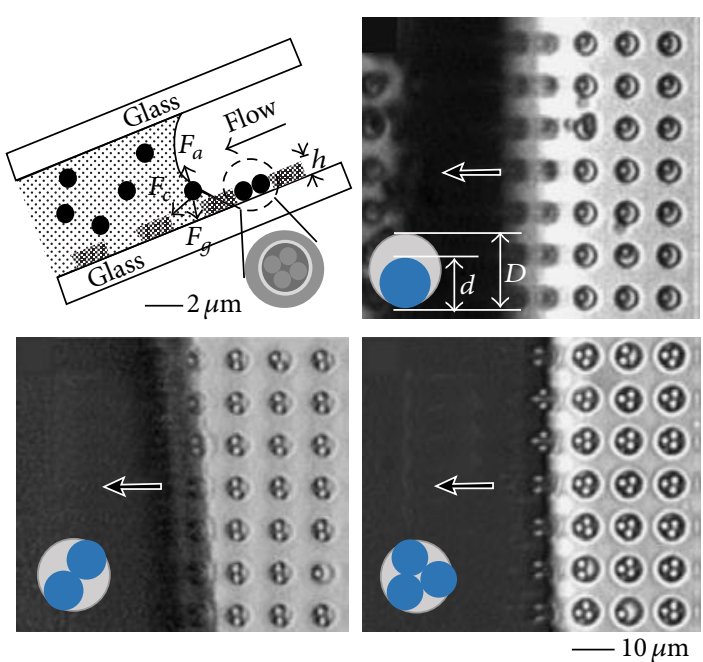

(a)

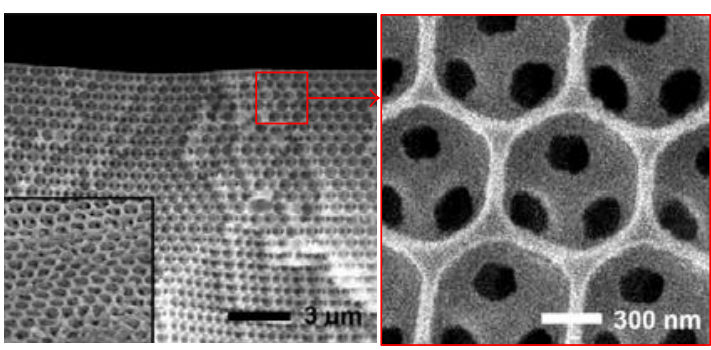

(c)

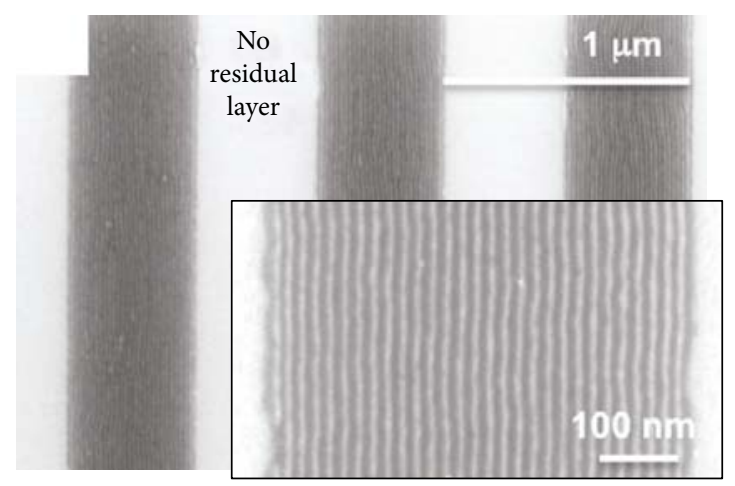

(e)

PS-b-PMMA
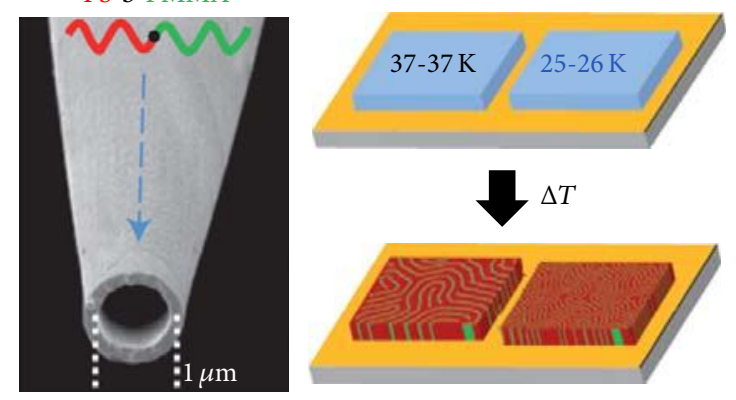

(g)

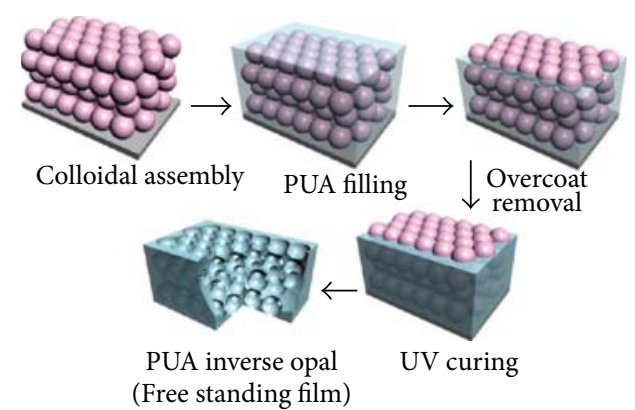

(b)

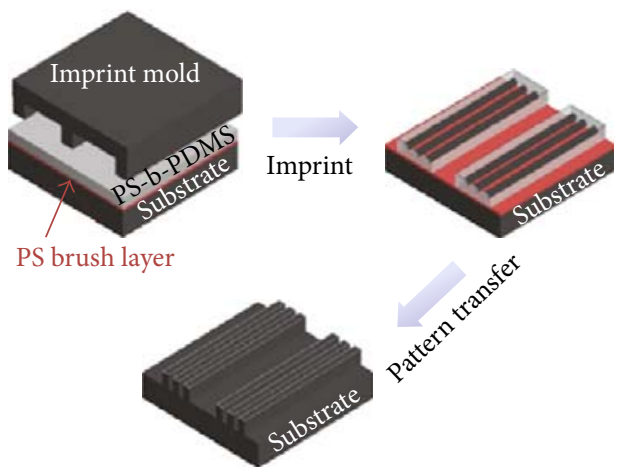

(d)

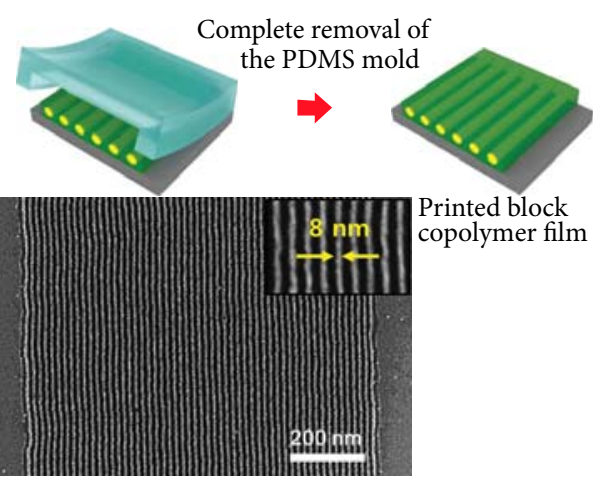

(f)

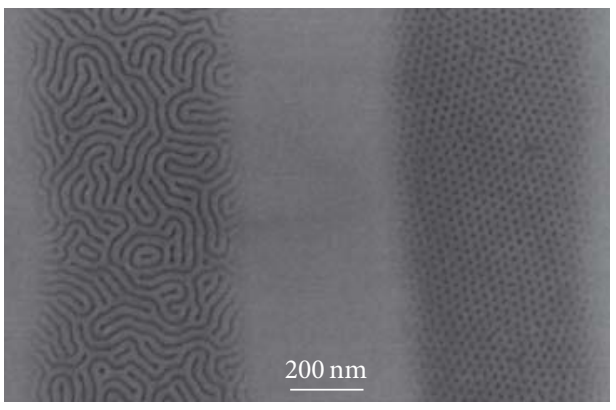

(h)

FIgure 4: Continued. 

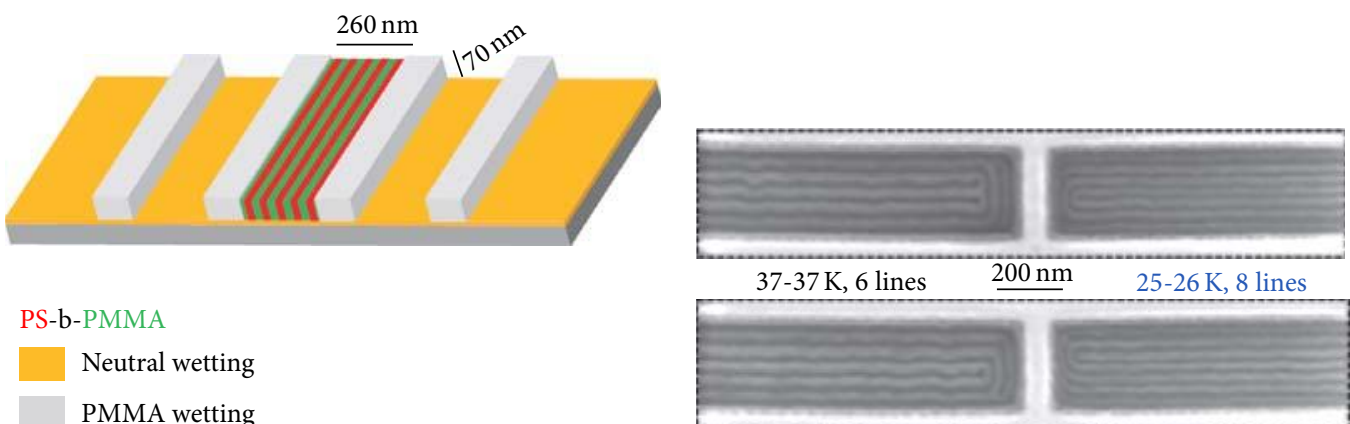

37-37 K, 6 lines $\quad 200 \mathrm{~nm} \quad 25-26 \mathrm{~K}, 8$ lines

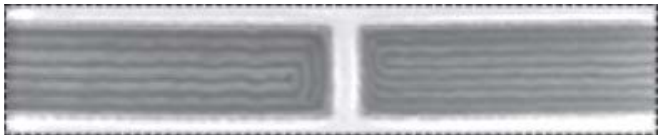

(i)

(j)

FIGURE 4: Integrated assembly with merged top-down with bottom-up approaches. (a) Schematic illustration of procedure for templateassisted self-assembly of spherical colloids (top left). Optical images show the assembly of various PS beads in an array of cylindrical holes $(3.1 \mu \mathrm{m}$ PS beads in cylindrical holes $5 \mu \mathrm{m}$ in diameter, top right; $2.5 \mu \mathrm{m}$ PS beads in cylindrical holes $5 \mu \mathrm{m}$ in diameter, top right; and $2.5 \mu \mathrm{m}$ PS beads in cylindrical holes $6 \mu \mathrm{m}$ in diameter, bottom right). The inset illustration indicates assembled structures (the diameter $(D)$ of holes and the diameter $(d)$ of spherical colloids). Reproduced with permission from [109]. Copyright 2003 WILEY-VCH Verlag $\mathrm{GmbH} \&$ Co. KGaA. (b) Schematic illustration of procedure for fabricating free-standing three-dimensional inverse opal (3D-IO) structure of polyurethane acrylate (PUA). (c) Cross-sectional (left) and top-view (right) SEM images of well-ordered 3D-IO structure of PUA. Reproduced with permission from [112]. Copyright 2012 American Chemical Society. (d) Schematic illustration of process for Sub-10 nm pattern transfer from self-assembled PS-b-PDMS with cylindrical patterns. Thermal nanoimprint induces the alignment of cylindrical BCP domains along the length direction of mold patterns. (e) SEM images showing the PDMS cylinders show no evidence of residual layer after plasma etching. The magnified SEM image shows the PDMS cylinders aligned along the long axis of the imprint grating. Reproduced with permission from [122]. Copyright 2011 American Chemical Society. (f) Schematic illustration of procedure for the sub-10 nm transfer printing of block copolymer self-assembly patterns. The inset SEM image shows the patterns after transfer printing on a bare Si substrate and treatment with oxidative plasma. Reproduced with permission from [123]. Copyright 2012 WILEY-VCH Verlag GmbH \& Co. KGaA. (g) SEM (left) image shows the tip of a glass capillary nozzle coated with $\mathrm{Au} / \mathrm{Pd}$. Thermal annealing $\left(220^{\circ} \mathrm{C}\right.$ for $\left.5 \mathrm{~min}\right)$ results in the self-assembled PS-b-PMMA BCPs on a substrate (right). (h) SEM image shows the self-assembled PS-b-PMMA BCPs with two different morphologies (lamellae form with MWs of 37-37 K, left; cylinder form with 46-21 K, right) printed as lines. (i) Schematic illustration of process for directed self-assembly of PS-b-PMMA BCPs printed onto topographically patterned substrate. (j) SEM images show the directed self-assembly of PS-b-PMMA BCPs with MWs of $37-37 \mathrm{~K}$ (left) and 25-26 K (right) in adjacent trenches (depth $\sim 70 \mathrm{~nm}$, width $\sim 260 \mathrm{~nm}$ ). Reproduced with permission from [124]. Copyright 2013 Macmillan Publishers Ltd.

monodispersed spherical colloids. The template can be fabricated by photolithography and either polystyrene (PS) beads or silica spheres were used as the spherical colloids. As illustrated in Figure 4(a), the template-assisted self-assembly process is influenced by three major forces: (i) the capillary force associated with the meniscus of the liquid slug, (ii) the gravitational force associated with the density difference between the particle and the dispersion solvent, and (iii) the electrostatic force caused by charges on the surfaces of the particles and the template. In particular, the capillary force plays a significant role in pushing particles into the template holes, taking excess particles along in the direction of the liquid slug. The yield of this assembly process depends on the balance of these three forces. By controlling the forces, the colloidal particles can be trapped in the template holes. The three optical micrographs in Figure 4(a) show a few examples of the single-layered and well-defined aggregates of colloidal particles during the in situ observation. Here, this method makes it possible to control the structural assemblies by the ratio between the diameter $(D)$ of holes and the diameter (d) of spherical colloids, ranging from $D / d=1.00$ to 3.30 . Furthermore, it might be useful to other systems such as cells $[110,111]$.
5.2. Colloidal Template Assisted for Fabrication 3D Porous Inverse Opal Structure. Yeo et al. [112] demonstrated an advanced tool of LbL coating of polyelectrolyte multilayers inside 3-dimensional porous inverse opal (3D-IO) templates. Uniformly deposited LbL films could be grown inside the 3DIO structures since the structures were free-standing with a double-sided open porosity formed by colloidal assembly and selective removal of the colloids. As shown in Figure 4(b), UV-curable polyurethane acrylate (PUA) prepolymer filled the voids between particles that were self-assembled into $3 \mathrm{D}$ opal structure in order to prepare the free-standing 3DIO structures. After curing PUA by flood UV exposure, the colloidal particles were removed by solvent, and freestanding 3D-IO structures were mechanically peeled from the sacrificial substrate. However, the bottom side spontaneously offered surface porosity because of contact between the substrate and the colloidal particle, whereas the topside pores were blocked by overcoated excess PUA. An ethanol solution was spin-coated on the overcoated layer several times because the shear-slip action of ethanol during spin-coating can physically remove the residual PUA. Figure 4 (c) shows the successfully fabricated 3D-IO PUA structure, wherein the top surface pores are completely opened. As a result, 
the LbL treated 3D-IO structures are used as nanofiltration membranes for removal of diluted copper ions and they show excellent membrane performance because of the nanoscale pores integrated in microscale inverse opal structures.

5.3. Nanoimprint Directed Self-Assembly of Block Copolymers. Directed self-assembly (DSA) of block copolymers (BCPs) has focused on promising nanolithography to realize highresolution nanopatterning with a typical feature size in the $3-50 \mathrm{~nm}$ region $[56,113,114]$. Generally, there are two approaches for DSA: (i) chemically [115-117] and (ii) topographically [118-121] patterned substrates. These approaches are useful but require high-cost and time-consuming processes for lithographic prepatterning of each substrate. Recently, Park et al. [122] presented an integrated assembly approach for the nanoimprint DSA of BCPs to address these shortcomings. The process for nanoimprint DSA of PS-bPDMS BCP is shown in Figure 4(d). In particular, the PS brush layer, which is deposited on the substrate, plays a significant role in attracting the majority of PS domains since bare silicon or silicon dioxide attracts a PDMS layer. After forming self-assembled BCPs via thermal imprint and release, the aligned PDMS cylinders are used as a template or etch-mask for sub-10 nm pattern transfer on the underlying substrate. As shown in Figure 4(e), after a plasma etching step, perfectly aligned BCP patterns were obtained along the long axis of the grating pattern without a residual layer.

5.4. NTP with Directed Self-Assembly of BCPs. Another interesting approach for integrating transfer printing and selfassembly BCPs was demonstrated by Jeong et al. [123] The process uses PDMS molds for directing the self-assembly of copolymers. The polydimethyl siloxane (PDMS) mold was prepared from a silicon master patterned with trenches with a width of $250 \mathrm{~nm}$ and a depth of $40 \mathrm{~nm}$ with a periodicity of $1.25 \mu \mathrm{m}$. In the next step, PS-b-PDMS films were spincoated on the PDMS mold and the mold was placed in a solvent annealing chamber containing acetone and toluene to form self-assembled BCPs. The PDMS mold coated with selfassembled BCPs was placed on the target substrate, and mild pressure of $\sim 20 \mathrm{kPa}$ was applied to transfer the self-assembled BCPs. Finally, after the transfer of the self-assembled BCP pattern, the elastomeric mold was removed from the substrate (Figure 4(f)). The inset SEM image shows that this approach can realize sub-10 $\mathrm{nm}$ transfer printing of BCPs on the Si substrate.

5.5. Self-Assembly of BCPs via High-Resolution Patterning Electrohydrodynamic Jet Printing. With increasing demand for nanoscale device applications, self-assembled BCPs have received a great deal of research attention as a means to the nanofabrication. However, rapid and high resolution patterning of multiple scale BCPs with diverse molecular weights (MWs) and composition is still challenging. Onses et al. [124] demonstrated a creative technique for self-assembling multiple BCPs via electrohydrodynamic jet (e-jet) printing on the same surface. E-jet printing that requires applying electric field during the jet printing process is possible to create complex patterns with different conductive inks such as conductive polymers, Si nanoparticles, or rods, and singlewalled carbon nanotubes [125]. Here, applied electric fields drive the flow of inks based on PS-b-PMMA BCPs with different MWs through nozzle tips. Thermal annealing then induces phase separation of the BCPs on the same substrate (Figure 4(g)). Figure 4(h) shows successful high-resolution printed BCPs with two different MWs. Moreover, the authors presented that the printing is useful to direct self-assembly of BCPs based on surface topography (Figure 4(i)). As shown in Figure 4(j), by using two BCP inks with different MWs, it is possible to generate the BCP domain with two different periodicities within the same trench area.

\section{Applications with Integrating Assembly}

The best advantage of integrating assembly lithographic involves the possibility to adjust the desired physical and chemical properties of fabricated samples by changing the feature size, thickness, and applied materials. In this section, we introduce several applications as examples of integrating assembly: photoluminescent microtags, graphene nanoribbon transistors, and multiscale porous nanocolander networks (Figure 5).

6.1. Colloid-Based Photoluminescent Microtags. Recently, Ressier et al. [126] reported a new patterning technique called electrical-NIL (e-NIL) for topographic and electrostatic imprinting of thermoplastic electret films at the nanoscale. The idea stems from electrical microcontact printing (e$\mu \mathrm{CP}$ ) which can selectively obtain chemical patterning having a surface charge gradient $[127,128]$. E-NIL consists of three steps: (i) a patterned conductive mold is pressed on the thermoplastic electret film above its glass transition temperature $T_{g}$, (ii) after cooling the temperature below its $T_{g}$, an electric field is applied between the mold and the substrate, and (iii) the mold is removed. Through e-NIL, it is possible to fabricate topographic and electrostatic patterns on desired spots. These patterns allow opportunities for directed assembly of colloidal nanoparticles on desired surfaces.

Diaz et al. [129] realized micron-sized photoluminescent quick response $(\mathrm{QR})$ codes which applied to directed electrostatic assembly of $28 \mathrm{~nm}$ upconverting lanthanide-doped $\beta$ $\mathrm{NaYF}_{4}$ nanocrystals inside e-NIL patterns. Figure 5(a) shows the transfer procedure of nanocrystals-based QR codes. First, PMMA was chosen as the thermoplastic electret due to its excellent charge storage properties and the capability to be readily imprinted by T-NIL [130-132]. The glue is cured by UV exposure, allowing nanocrystals to bind to e-NIL patterns, and then immersed in a water bath to remove the polyvinyl acetate (PVA) sacrificial layer. These $\mathrm{NaYF}_{4}$ nanocrystal-based QR codes were transferred to an adhesive polyethylene terephthalate (PET) film and the thin film can be used as a sticker to tag desired products. Also, after black and white light conversion and inversion of optical microscopy image of photoluminescence (PL) mapping at $545 \mathrm{~nm}$, the 50 micron-sized nanocrystal-based QR 


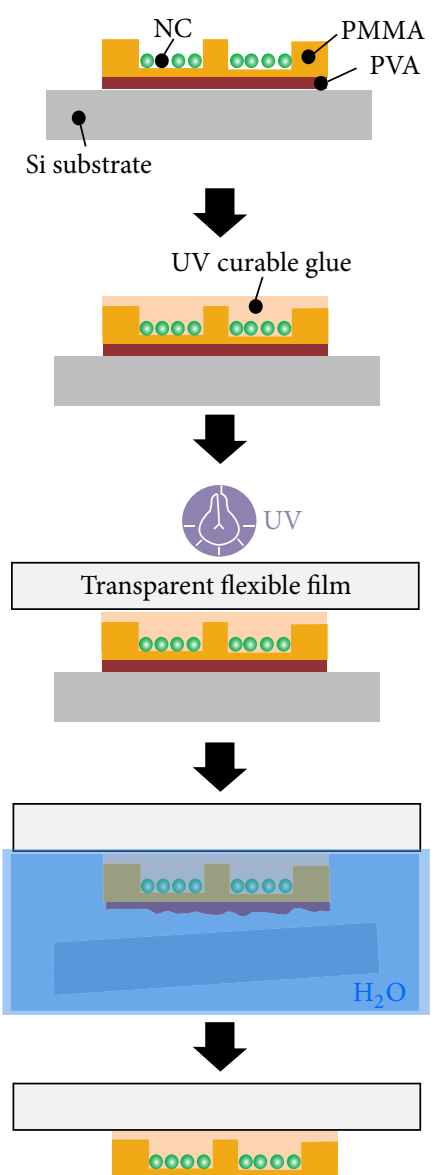

(a)

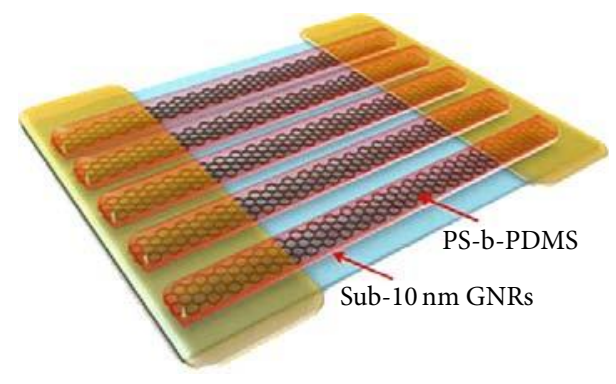

(c)

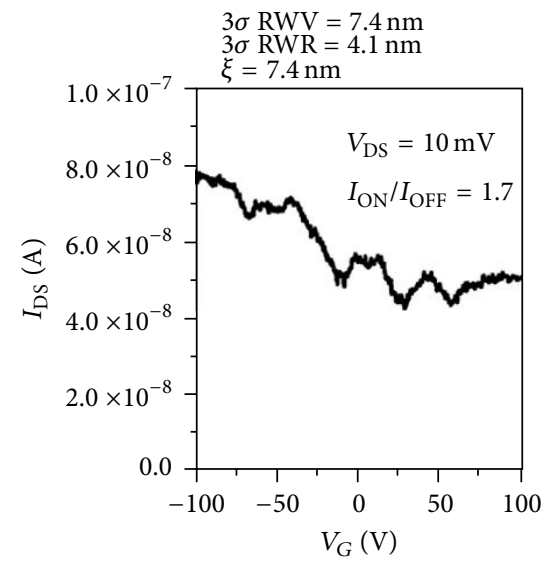

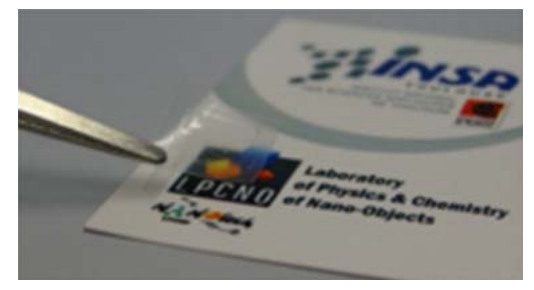
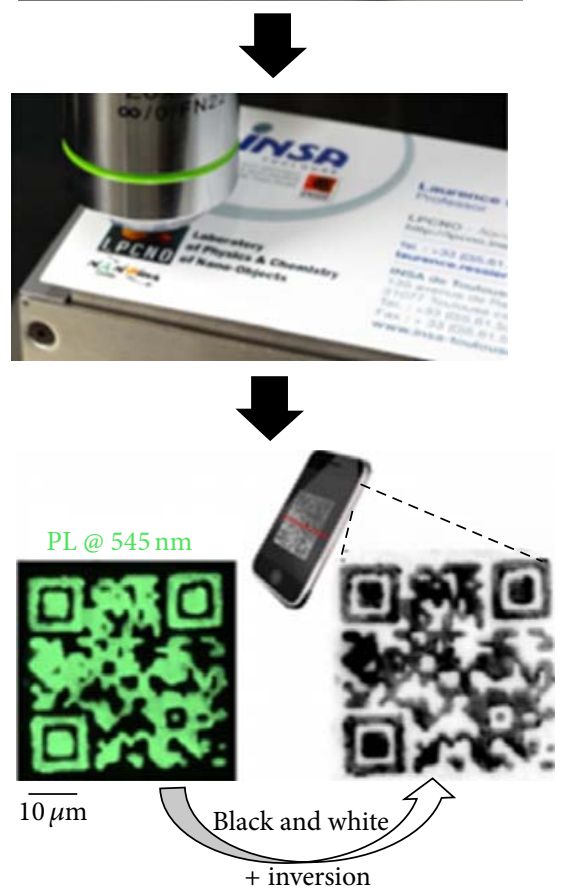

(b)

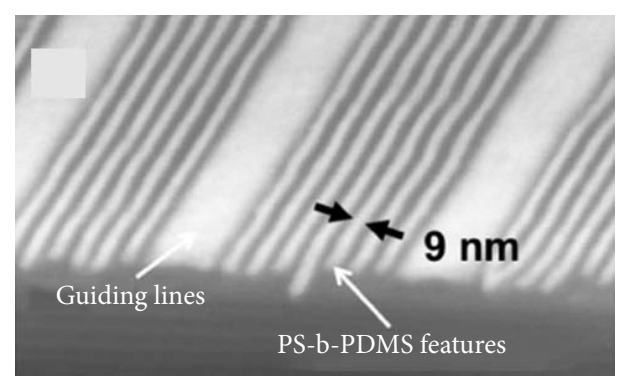

(d)

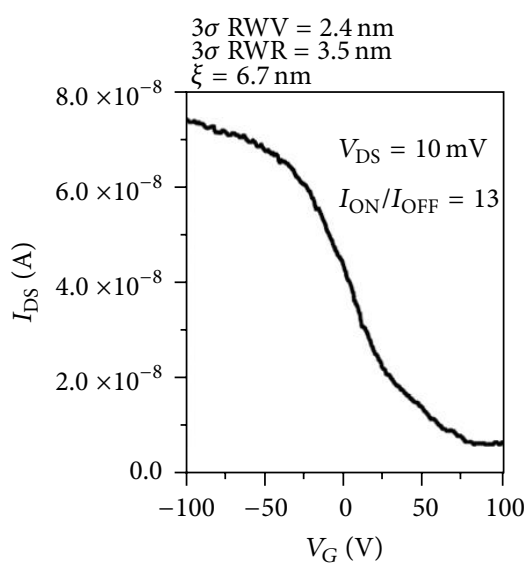

(e)

Figure 5: Continued. 

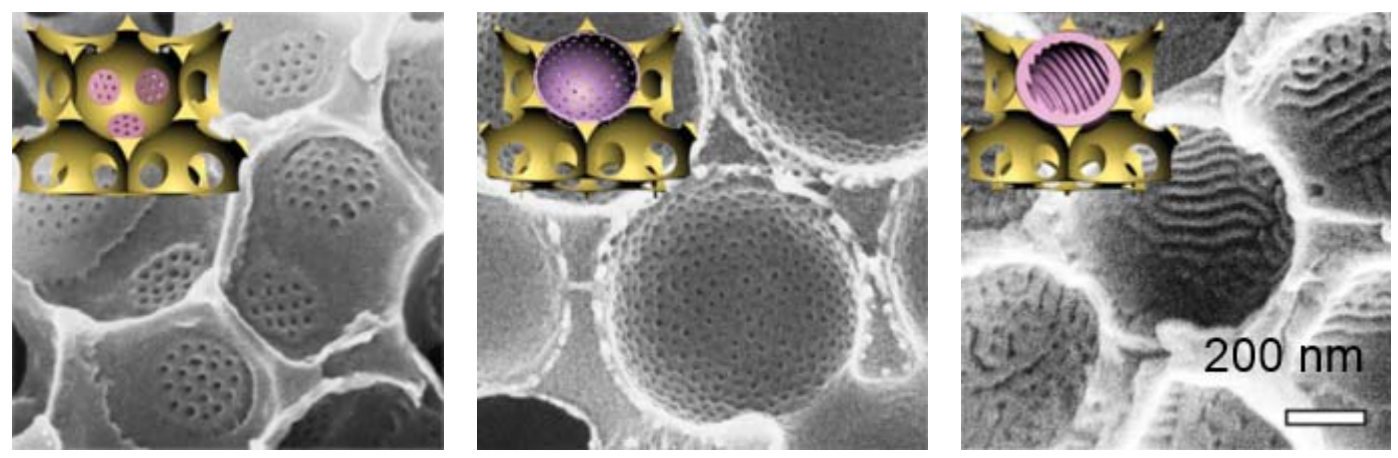

(f)

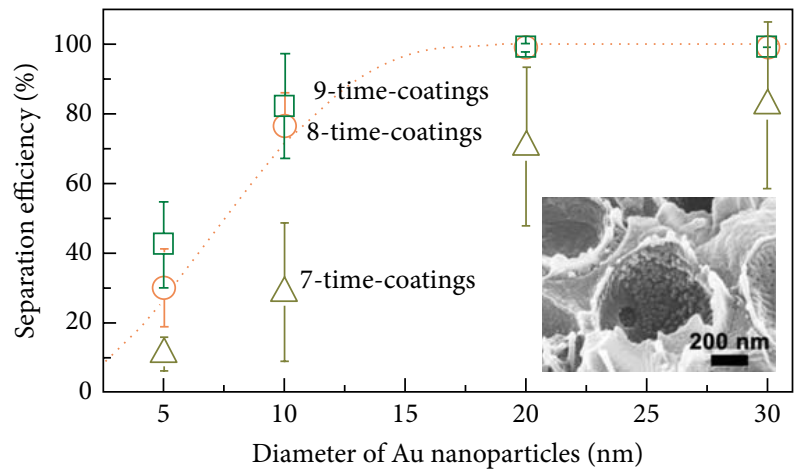

(g)

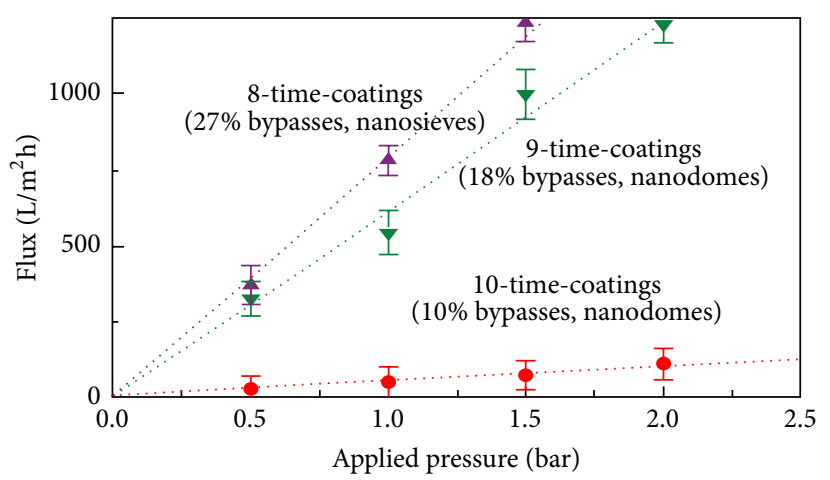

(h)

FIGURE 5: Applications of 3D hierarchical structures fabricated by integrated assembly with merged top-down with bottom-up approaches. (a-b) (a) Schematic illustration for the transfer procedure for developed QR code stickers that could be laminated on target products. (b) An example of a $1 \mathrm{~cm}^{2}$ sticker containing one thousand $50 \mu \mathrm{m}$ sized QR codes made of $28 \mathrm{~nm} \beta-\mathrm{NaYF}_{4}$ nanocrystals, integrated on a private official document (top) and optical microscopy image of the PL mapping at $545 \mathrm{~nm}$ of one of the QR codes (middle). After black and white conversion and inversion of the image, the $50 \mu \mathrm{m}$ NC-QR code, which directs to the internet link, is readable using an appropriate smartphone application (bottom). Reproduced with permission from [129]. Copyright 2014 IOP Publishing Ltd. (c-e) (c) Conceptual illustration for the multichannel transistor with densely aligned sub-10 nm graphene nanoribbons. (d) SEM image showing well-oriented $9 \mathrm{~nm}$ half-pitch PDMS cylinders that were revealed from the segregated PS-b-PDMS film by $\mathrm{O}_{2}$ plasma etching. (e) $I_{\mathrm{DS}}-V_{G}$ characteristic curves of the three multichannel FETs with ribbon-to-ribbon width variation of $7.4 \mathrm{~nm}$ (left), $5.1 \mathrm{~nm}$ (middle), and $2.4 \mathrm{~nm}$ (right). Reproduced with permission from [145]. Copyright 2012 American Chemical Society. (f-h) (f) SEM images show morphology control of the BCP thin films inside the hollow chamber of the inverse opal (IO) frame according to the BCP film thickness. Inset illustration depicts structures ranging from nanosieves (left) to nanodomes (middle) and corrugated structures containing both perpendicular and parallel cylinders (right). (g) Size-selective separation performance of the 3D nanocolander membrane with varying particulate size of Au nanoparticles ( 5 to $30 \mathrm{~nm}$ ). The pore size of the BCP nanosieve was fixed at $18 \mathrm{~nm}$ in diameter (corresponding to the volume fraction of PMMA at 28\%). The dotted line represents the estimated separated efficiency of filtration with $18 \mathrm{~nm}$-sized pores. (h) Plot of pure water flux through membranes with respect to the operating pressure with varying numbers of BCP solution coatings. Reproduced with permission from [153]. Copyright 2014 WILEY-VCH Verlag GmbH \& Co. KGaA.

code can be read with an available smartphone application (Figure 5(b)). Beyond this application, combining localized charge injection through e-NIL with other charged or polarizable colloidal particles has great potentials for colloid-based devices and sensors.

\subsection{Fabrication of Sub-10 $\mathrm{nm}$ Scale Graphene Nanoribbon} Transistor by BCP Lithography. Graphene has remarkable electronic and mechanical properties [133-136]. However, field-effect transistors (FETs) based on graphene show poor on/off current ratios because graphene is a zero-bandgap semiconductor [137]. In order to open up the bandgap, graphene nanostructures, such as graphene nanoribbons
(GNRs) $[138,139]$ and graphene nanomeshes (GNMs) [140142] have been extensively studied. In particular, to create densely aligned arrays of sub-10 nm wide graphene nanostructures over a large area, block copolymer (BCP) selfassembly in combination with nanoimprint lithography is a promising lithographic technique [122, 143-145].

Recently, Liang and Wi [145] showed FETs consisting of densely arranged GNRs (the total number of GNR channels in a FET is approximately 50) made by a nanofabrication method (sub-10 nm) that merges NIL and directed selfassembly of BCPs (Figures 5(c) and 5(d)). Furthermore, they showed that the on/off current ratio of the FETs bearing such GNRs is significantly affected by ribbon-to-ribbon 
width variation (RWV) of multiple GNRs depending on the processing conditions of BCP self-assembly. As shown in Figure 5(e), a relatively large RWV among GNRs results in a lower on/off current ratio, which is caused by the nonsynchronization of the off states of multiple nonuniform GNRs. In particular, the $8 \mathrm{~nm}$ half-pitch GNRs-based FET with RWV of less than $3 \mathrm{~nm}$ exhibits a high on/off current ratio $(>10)$, which is of higher value than reported transistors bearing densely aligned GNRs [146]. This research offers crucial insights for understanding the transport characteristics of the FETs based on multiple GNRs, which may lead to high resolution graphene nanostructures for future electronic applications.

6.3. Multiscale Porous Nanocolander Network with Tunable Transport Properties by Combined CL with LbL Processes. 3D inverse opal (IO) structure, which is constructed from the self-assembled colloidal particles, is an attractive structure for various applications such as photonic crystals [147], optical biosensors [148], and energy devices [149-152]. Previous researches take advantage of large interfacial area and highly periodic structures generally have morphological benefits including high open porosity. Nevertheless, systematic control of permeability and selectivity of porous media is challenging because the size of colloids used for each templating must change.

Kim et al. [153] presented an interesting approach for a multiscale porous network with tunable transport properties. The microphase-separated block copolymer creates welldefined mesopores inside the 3D-IO structured template that was obtained from a UV-curable polymer, PUA. According to the increasing numbers of BCP solution coating, the film thickness is increased and BCP morphology is changed from nanosieves to nanodomes and corrugated structures (Figure 5(f)). The multiscale porous networks were tested for membrane separation performance with Au nanoparticle solutions (the pore size of the BCP nanosieve was fixed at $18 \mathrm{~nm}$ and $\mathrm{Au}$ nanoparticle solution contained particles with diameters of $5,10,20$, or $30 \mathrm{~nm}$ ) (Figure $5(\mathrm{~g})$ ). The results show complete separation of the nanoparticles, when the number of BCP solution coatings is greater than 8 without forming bulk-scale defects. Also the value of water permeability for testing the transport performance is higher than other presented BCP-based membranes (Figure 5(h)) [154-156]. Therefore, multiscale porous network structures with tunable transport properties can serve as a significant platform for filtration applications.

\section{Conclusion}

In this review, advanced methods in unconventional lithography for 3D hierarchical nanostructures are described. These methods can produce structures that can be used for many applications that can complement those already available from conventional nanolithography or nanotechnology. The range of the 3D nanostructures and techniques can create an appealing set of circumstances for development of uses for basic science and industry in the near future.

\section{Conflict of Interests}

The authors declare that there is no conflict of interests regarding the publication of this paper.

\section{Acknowledgments}

This work is supported by the IBS-R015-D1, Basic Science Research Program through the National Research Foundation of Korea funded by the Ministry of Science, ICT and Future Planning (NRF-2013-R1A1A1061403), and the Pioneer Research Center Program (NRF-2014M3C1A3001208).

\section{References}

[1] H. J. Levinson, Principles of Lithography, SPIE, Bellingham, Wash, USA, 2005.

[2] Y. Xia and G. Whitesides, "Soft lithography," Angewandte Chemie-International Edition, vol. 37, pp. 550-575, 1998.

[3] Y. Xia, J. A. Rogers, K. E. Paul, and G. M. Whitesides, "Unconventional methods for fabricating and patterning nanostructures," Chemical Reviews, vol. 99, no. 7, pp. 1823-1848, 1999.

[4] B. D. Gates, Q. Xu, J. C. Love, D. B. Wolfe, and G. M. Whitesides, "Unconventional nanofabrication," Annual Review of Materials Research, vol. 34, pp. 339-372, 2004.

[5] D. G. Bucknall, Nanolithography and Patterning Techniques in Microelectronics, CRC Press, Boca Raton, Fla, USA, 2005.

[6] B. D. Gates, Q. Xu, M. Stewart, D. Ryan, C. G. Willson, and G. M. Whitesides, "New approaches to nanofabrication: molding, printing, and other techniques," Chemical Reviews, vol. 105, no. 4, pp. 1171-1196, 2005.

[7] S. Y. Chou, P. R. Krauss, and P. J. Renstrom, "Imprint of sub$25 \mathrm{~nm}$ vias and trenches in polymers," Applied Physics Letters, vol. 67, pp. 3114-3116, 1995.

[8] K. Y. Suh, Y. S. Kim, and H. H. Lee, "Capillary force lithography," Advanced Materials, vol. 13, no. 18, pp. 1386-1389, 2001.

[9] K. Y. Suh and H. H. Lee, "Capillary force lithography: largearea patterning, self-organization, and anisotropic dewetting," Advanced Functional Materials, vol. 12, no. 6-7, pp. 405-413, 2002.

[10] R. D. Piner, J. Zhu, F. Xu, S. Hong, and C. A. Mirkin, “'Dip-pen' nanolithography," Science, vol. 283, no. 5402, pp. 661-663, 1999.

[11] Y. S. Kim, K. Y. Suh, and H. H. Lee, "Fabrication of threedimensional microstructures by soft molding," Applied Physics Letters, vol. 79, no. 14, pp. 2285-2287, 2001.

[12] Y. S. Kim, J. Park, and H. H. Lee, "Three-dimensional pattern transfer and nanolithography: modified soft molding," Applied Physics Letters, vol. 81, no. 6, pp. 1011-1013, 2002.

[13] A. Perl, D. N. Reinhoudt, and J. Huskens, "Microcontact printing: limitations and achievements," Advanced Materials, vol. 21, no. 22, pp. 2257-2268, 2009.

[14] S.-M. Yang, S. G. Jang, D.-G. Choi, S. Kim, and H. K. Yu, "Nanomachining by colloidal lithography," Small, vol. 2, no. 4, pp. 458-475, 2006.

[15] M. Park, C. Harrison, P. M. Chaikin, R. A. Register, and D. H. Adamson, "Block copolymer lithography: periodic arrays of $10^{11}$ holes in 1 square centimeter," Science, vol. 276, no. 5317, pp. 1401-1404, 1997.

[16] J. A. Rogers and H. H. Lee, Unconventional Nanopatterning Techniques and Applications, John Wiley \& Sons, Hoboken, NJ, USA, 2009. 
[17] H. E. Jeong, R. Kwak, A. Khademhosseini, and K. Y. Suh, "UVassisted capillary force lithography for engineering biomimetic multiscale hierarchical structures: from lotus leaf to gecko foot hairs," Nanoscale, vol. 1, no. 3, pp. 331-338, 2009.

[18] H. E. Jeong, S. H. Lee, J. K. Kim, and K. Y. Suh, "Nanoengineered multiscale hierarchical structures with tailored wetting properties," Langmuir, vol. 22, no. 4, pp. 1640-1645, 2006.

[19] W.-G. Bae, H. N. Kim, D. Kim, S.-H. Park, H. E. Jeong, and K.Y. Suh, "25th anniversary article: scalable multiscale patterned structures inspired by nature: the role of hierarchy," Advanced Materials, vol. 26, no. 5, pp. 675-699, 2014.

[20] L. J. Guo, "Recent progress in nanoimprint technology and its applications," Journal of Physics D. Applied Physics, vol. 37, no. 11, pp. R123-R141, 2004.

[21] L. J. Guo, "Nanoimprint lithography: methods and material requirements," Advanced Materials, vol. 19, no. 4, pp. 495-513, 2007.

[22] S. Y. Chou, P. R. Krauss, and P. J. Renstrom, "Imprint lithography with 25-nanometer resolution," Science, vol. 272, no. 5258, pp. 85-87, 1996.

[23] S. Y. Chou, P. R. Krauss, and P. J. Renstrom, "Nanoimprint lithography," Journal of Vacuum Science and Technology B: Microelectronics and Nanometer Structures, vol. 14, no. 6, pp. 4129-4133, 1996.

[24] M. Colburn, S. C. Johnson, M. D. Stewart et al., "Step and flash imprint lithography: a new approach to high-resolution patterning," in Emerging Lithographic Technologies III, vol. 3676 of Proceedings of SPIE, pp. 379-389, 1999.

[25] S.-M. Seo, T.-I. Kim, and H. H. Lee, "Simple fabrication of nanostructure by continuous rigiflex imprinting," Microelectronic Engineering, vol. 84, no. 4, pp. 567-572, 2007.

[26] S. H. Ahn and L. J. Guo, "High-speed roll-to-roll nanoimprint lithography on flexible plastic substrates," Advanced Materials, vol. 20, no. 11, pp. 2044-2049, 2008.

[27] S. H. Ahn and L. J. Guo, "Large-area roll-to-roll and roll-toplate Nanoimprint Lithography: a step toward high-throughput application of continuous nanoimprinting," ACS Nano, vol. 3, no. 8, pp. 2304-2310, 2009.

[28] T. Haatainen and J. Ahopelto, "Pattern transfer using step\&stamp imprint lithography," Physica Scripta, vol. 67, no. 4, pp. 357-360, 2003.

[29] K. Y. Suh, P. Kim, and H. H. Lee, "Capillary kinetics of thin polymer films in permeable microcavities," Applied Physics Letters, vol. 85, no. 18, pp. 4019-4021, 2004.

[30] D. Suh, H. Tak, S.-J. Choi, and T.-I. Kim, "Permeabilityand surface-energy-tunable polyurethane acrylate molds for capillary force lithography," ACS Applied Materials \& Interfaces, vol. 7, no. 43, pp. 23824-23830, 2015.

[31] H. Yoon, T.-I. Kim, S. Choi, K. Y. Suh, M. J. Kim, and H. H. Lee, "Capillary force lithography with impermeable molds," Applied Physics Letters, vol. 88, Article ID 254104, 2006.

[32] J. Zaumseil, M. A. Meitl, J. W. P. Hsu et al., "Three-dimensional and multilayer nanostructures formed by nanotransfer printing," Nano Letters, vol. 3, no. 9, pp. 1223-1227, 2003.

[33] A. Carlson, A. M. Bowen, Y. Huang, R. G. Nuzzo, and J. A. Rogers, "Transfer printing techniques for materials assembly and micro/nanodevice fabrication," Advanced Materials, vol. 24, no. 39, pp. 5284-5318, 2012.

[34] M. A. Meitl, Z.-T. Zhu, V. Kumar et al., "Transfer printing by kinetic control of adhesion to an elastomeric stamp," Nature Materials, vol. 5, no. 1, pp. 33-38, 2006.
[35] T.-I. Kim, J.-H. Kim, S. J. Son, and S.-M. Seo, “Gold nanocones fabricated by nanotransfer printing and their application for field emission," Nanotechnology, vol. 19, no. 29, Article ID 295302, 2008.

[36] T.-I. Kim and S.-M. Seo, "The facile fabrication of a wire-grid polarizer by reversal rigiflex printing," Nanotechnology, vol. 20, no. 14, Article ID 145305, 2009.

[37] M. K. Kwak, T.-I. Kim, P. Kim, H. H. Lee, and K. Y. Suh, "Largearea dual-scale metal transfer by adhesive force," Small, vol. 5, no. 8, pp. 928-932, 2009.

[38] Y. S. Kim, S. J. Baek, and P. T. Hammond, "Physical and chemical nanostructure transfer in polymer spin-transfer printing," Advanced Materials, vol. 16, no. 7, pp. 581-584, 2004.

[39] D. Suh, S.-J. Choi, and H. H. Lee, "Rigiflex lithography for nanostructure transfer," Advanced Materials, vol. 17, no. 12, pp. 1554-1560, 2005.

[40] M. H. Lee, J. Y. Lin, and T. W. Odom, "Large-area nanocontact printing with metallic nanostencil masks," Angewandte Chemie-International Edition, vol. 49, no. 17, pp. 3057-3060, 2010.

[41] E. Menard, K. J. Lee, D.-Y. Khang, R. G. Nuzzo, and J. A. Rogers, "A printable form of silicon for high performance thin film transistors on plastic substrates," Applied Physics Letters, vol. 84, no. 26, pp. 5398-5400, 2004.

[42] D.-H. Kim, J.-H. Ahn, M. C. Won et al., "Stretchable and foldable silicon integrated circuits," Science, vol. 320, no. 5875, pp. 507-511, 2008.

[43] T.-H. Kim, A. Carlson, J.-H. Ahn et al., "Erratum: 'kinetically controlled, adhesiveless transfer printing using microstructured stamps' [Appl. Phys. Lett.94, 113502 (2009)]," Applied Physics Letters, vol. 94, Article ID 189902, 2009.

[44] T.-I. Kim, R.-H. Kim, and J. A. Rogers, "Microscale inorganic light-emitting diodes on flexible and stretchable substrates," IEEE Photonics Journal, vol. 4, no. 2, pp. 607-612, 2012.

[45] T.-I. Kim, Y. H. Jung, J. Song et al., "High-efficiency, microscale GaN light-emitting diodes and their thermal properties on unusual substrates," Small, vol. 8, no. 11, pp. 1643-1649, 2012.

[46] R.-H. Kim, H. Tao, T.-I. Kim et al., "Materials and designs for wirelessly powered implantable light-emitting systems," Small, vol. 8, no. 18, pp. 2812-2818, 2012.

[47] R.-H. Kim, S. Kim, Y. M. Song et al., "Flexible vertical light emitting diodes," Small, vol. 8, no. 20, pp. 3123-3128, 2012.

[48] T.-I. Kim, J. G. McCall, Y. H. Jung et al., "Injectable, cellularscale optoelectronics with applications for wireless optogenetics," Science, vol. 340, no. 6129, pp. 211-216, 2013.

[49] J.-W. Jeong, J. G. Mccall, G. Shin et al., "Wireless optofluidic systems for programmable in vivo pharmacology and optogenetics," Cell, vol. 162, no. 3, pp. 662-674, 2015.

[50] D.-H. Kim, N. Lu, R. Ghaffari et al., "Materials for multifunctional balloon catheters with capabilities in cardiac electrophysiological mapping and ablation therapy," Nature Materials, vol. 10, no. 4, pp. 316-323, 2011.

[51] C. L. Cheung, R. J. Nikolić, C. E. Reinhardt, and T. F. Wang, "Fabrication of nanopillars by nanosphere lithography," Nanotechnology, vol. 17, no. 5, pp. 1339-1343, 2006.

[52] Y. A. Vlasov, X.-Z. Bo, J. C. Sturm, and D. J. Norris, "On-chip natural assembly of silicon photonic bandgap crystals," Nature, vol. 414, no. 6861, pp. 289-293, 2001.

[53] J. H. Moon, S. Kim, G.-R. Yi, Y.-H. Lee, and S.-M. Yang, "Fabrication of ordered macroporous cylinders by colloidal templating in microcapillaries," Langmuir, vol. 20, no. 5, pp. 2033-2035, 2004. 
[54] J.-M. Lim, G.-R. Yi, J. H. Moon, C.-J. Heo, and S.-M. Yang, "Superhydrophobic films of electrospun fibers with multiplescale surface morphology," Langmuir, vol. 23, no. 15, pp. 79817989, 2007.

[55] Y. Li, N. Koshizaki, H. Wang, and Y. Shimizu, "Untraditional approach to complex hierarchical periodic arrays with trinary stepwise architectures of micro-, submicro-, and nanosized structures based on binary colloidal crystals and their fine structure enhanced properties," ACS Nano, vol. 5, no. 12, pp. 9403-9412, 2011.

[56] F. S. Bates and G. H. Fredrickson, "Block copolymer thermodynamics: theory and experiment," Annual Review of Physical Chemistry, vol. 41, no. 1, pp. 525-557, 1990.

[57] C. Park, J. Yoon, and E. L. Thomas, "Enabling nanotechnology with self assembled block copolymer patterns," Polymer, vol. 44, no. 22, pp. 6725-6760, 2003.

[58] C. M. Bates, M. J. Maher, D. W. Janes, C. J. Ellison, and C. G. Willson, "Block copolymer lithography," Macromolecules, vol. 47, no. 1, pp. 2-12, 2014.

[59] C. Greiner, E. Arzt, and A. del Campo, "Hierarchical gecko-like adhesives," Advanced Materials, vol. 21, no. 4, pp. 479-482, 2009.

[60] Y. Hirai, S. Yoshida, and N. Takagi, "Defect analysis in thermal nanoimprint lithography," Journal of Vacuum Science and Technology B, vol. 21, no. 6, pp. 2765-2770, 2003.

[61] F. Zhang and H. Y. Low, "Ordered three-dimensional hierarchical nanostructures by nanoimprint lithography," Nanotechnology, vol. 17, no. 8, pp. 1884-1890, 2006.

[62] H. Schift, "Nanoimprint lithography: an old story in modern times? A review," Journal of Vacuum Science and Technology B: Microelectronics and Nanometer Structures, vol. 26, no. 2, pp. 458-480, 2008.

[63] L.-R. Bao, X. Cheng, X. D. Huang, L. J. Guo, S. W. Pang, and A. F. Yee, "Nanoimprinting over topography and multilayer three-dimensional printing," Journal of Vacuum Science and Technology B, vol. 20, no. 6, pp. 2881-2886, 2002.

[64] N. Kehagias, V. Reboud, G. Chansin et al., "Submicron three-dimensional structures fabricated by reverse contact UV nanoimprint lithography," Journal of Vacuum Science \& Technology B, vol. 24, pp. 3002-3005, 2006.

[65] X.-M. Zhao, Y. Xia, and G. M. Whitesides, "Fabrication of three-dimensional micro-structures: microtransfer molding," Advanced Materials, vol. 8, no. 10, pp. 837-840, 1996.

[66] D. J. Lipomi, R. C. Chiechi, M. D. Dickey, and G. M. Whitesides, "Fabrication of conjugated polymer nanowires by edge lithography," Nano Letters, vol. 8, no. 7, pp. 2100-2105, 2008.

[67] C. M. Bruinink, M. Péter, P. A. Maury et al., "Capillary force lithography: fabrication of functional polymer templates as versatile tools for nanolithography," Advanced Functional Materials, vol. 16, no. 12, pp. 1555-1565, 2006.

[68] S. A. Harfenist, S. D. Cambron, E. W. Nelson et al., "Direct drawing of suspended filamentary micro- and nanostructures from liquid polymers," Nano Letters, vol. 4, no. 10, pp. 1931-1937, 2004.

[69] M. Yun, N. V. Myung, R. P. Vasquez, C. Lee, E. Menke, and R. M. Penner, "Electrochemically grown wires for individually addressable sensor arrays," Nano Letters, vol. 4, no. 3, pp. 419422, 2004.

[70] N. T. Kemp, D. McGrouther, J. W. Cochrane, and R. Newbury, "Bridging the gap: polymer nanowire devices," Advanced Materials, vol. 19, no. 18, pp. 2634-2638, 2007.
[71] R. Kwak, H. E. Jeong, and K. Y. Suh, "Fabrication of monolithic bridge structures by vacuum-assisted capillary-force lithography," Small, vol. 5, no. 7, pp. 790-794, 2009.

[72] S. Neuhaus, N. D. Spencer, and C. Padeste, "Anisotropic wetting of microstructured surfaces as a function of surface chemistry," ACS Applied Materials and Interfaces, vol. 4, no. 1, pp. 123-130, 2012.

[73] D. Xia and S. R. J. Brueck, "Strongly anisotropic wetting on surfaces 2008," Nano Letters, vol. 8, pp. 2819-2824, 2008.

[74] J. Y. Chung, J. P. Youngblood, and C. M. Stafford, "Anisotropic wetting on tunable micro-wrinkled surfaces," Soft Matter, vol. 3, no. 9, pp. 1163-1169, 2007.

[75] M. K. Kwak, H.-E. Jeong, T.-I. Kim, H. Yoon, and K. Y. Suh, "Bio-inspired slanted polymer nanohairs for anisotropic wetting and directional dry adhesion," Soft Matter, vol. 6, no. 9, pp. 1849-1857, 2010.

[76] Y. Zheng, X. Gao, and L. Jiang, "Directional adhesion of superhydrophobic butterfly wings," Soft Matter, vol. 3, no. 2, pp. 178-182, 2007.

[77] T.-I. Kim and K. Y. Suh, "Unidirectional wetting and spreading on stooped polymer nanohairs," Soft Matter, vol. 5, no. 21, pp. 4131-4135, 2009.

[78] J. Oeffner and G. V. Lauder, "The hydrodynamic function of shark skin and two biomimetic applications," Journal of Experimental Biology, vol. 215, no. 5, pp. 785-795, 2012.

[79] L. Feng, S. Li, Y. Li et al., "Super-hydrophobic surfaces: from natural to artificial," Advanced Materials, vol. 14, no. 24, pp. 1857-1860, 2002.

[80] Z. Guo, W. Liu, and B.-L. Su, "Superhydrophobic surfaces: from natural to biomimetic to functional," Journal of Colloid and Interface Science, vol. 353, no. 2, pp. 335-355, 2011.

[81] L. Zhai, M. C. Berg, F. Ç. Cebeci et al., "Patterned superhydrophobic surfaces: toward a synthetic mimic of the namib desert beetle," Nano Letters, vol. 6, no. 6, pp. 1213-1217, 2006.

[82] B. Radha, S. H. Lim, M. S. M. Saifullah, and G. U. Kulkarni, "Metal hierarchical patterning by direct nanoimprint lithography," Scientific Reports, vol. 3, article 1078, 8 pages, 2013.

[83] A. Y. Vorobyev and C. Guo, "Multifunctional surfaces produced by femtosecond laser pulses," Journal of Applied Physics, vol. 117, no. 3, Article ID 033103, 2015.

[84] H. E. Jeong, J.-K. Lee, H. N. Kim, S. H. Moon, and K. Y. Suh, "A nontransferring dry adhesive with hierarchical polymer nanohairs," Proceedings of the National Academy of Sciences of the United States of America, vol. 106, no. 14, pp. 5639-5644, 2009.

[85] S. Kim, J. Wu, A. Carlson et al., "Microstructured elastomeric surfaces with reversible adhesion and examples of their use in deterministic assembly by transfer printing," Proceedings of the National Academy of Sciences of the United States of America, vol. 107, no. 40, pp. 17095-17100, 2010.

[86] S. Kim, M. Spenko, S. Trujillo, B. Heyneman, D. Santos, and M. R. Cutkoskly, "Smooth vertical surface climbing with directional adhesion," IEEE Transactions on Robotics, vol. 24, no. 1, pp. 65-74, 2008.

[87] W. R. Hansen and K. Autumn, "Evidence for self-cleaning in gecko setae," Proceedings of the National Academy of Sciences of the United States of America, vol. 102, no. 2, pp. 385-389, 2005.

[88] J. Lee and R. S. Fearing, "Contact self-cleaning of synthetic gecko adhesive from polymer microfibers," Langmuir, vol. 24, no. 19, pp. 10587-10591, 2008. 
[89] S. Kim, E. Cheung, and M. Sitti, "Wet self-cleaning of biologically inspired elastomer mushroom shaped microfibrillar adhesives," Langmuir, vol. 25, no. 13, pp. 7196-7199, 2009.

[90] M. K. Kwak, H.-E. Jeong, and K. Y. Suh, "Rational design and enhanced biocompatibility of a dry adhesive medical skin patch," Advanced Materials, vol. 23, no. 34, pp. 3949-3953, 2011.

[91] W. G. Bae, D. Kim, M. K. Kwak, L. Ha, S. M. Kang, and K. Y. Suh, "Enhanced skin adhesive patch with modulus-tunable composite micropillars," Advanced Healthcare Materials, vol. 2, no. 1, pp. 109-113, 2013.

[92] C. Dekker, "Solid-state nanopores," Nature Nanotechnology, vol. 2, no. 4, pp. 209-215, 2007.

[93] C. C. Striemer, T. R. Gaborski, J. L. McGrath, and P. M. Fauchet, "Charge- and size-based separation of macromolecules using ultrathin silicon membranes," Nature, vol. 445, no. 7129, pp. 749-753, 2007.

[94] S. W. Kowalczyk, L. Kapinos, T. R. Blosser et al., "Singlemolecule transport across an individual biomimetic nuclear pore complex," Nature Nanotechnology, vol. 6, no. 7, pp. 433438, 2011.

[95] O. Vazquez-Mena, G. Villanueva, V. Savu, K. Sidler, M. A. F. van den Boogaart, and J. Brugger, "Metallic nanowires by full wafer stencil lithography," Nano Letters, vol. 8, no. 11, pp. 3675-3682, 2008.

[96] J. Henzie, M. H. Lee, and T. W. Odom, "Multiscale patterning of plasmonic metamaterials," Nature Nanotechnology, vol. 2, no. 9, pp. 549-554, 2007.

[97] S. Aksu, A. A. Yanik, R. Adato, A. Artar, M. Huang, and H. Altug, "High-throughput nanofabrication of infrared plasmonic nanoantenna arrays for vibrational nanospectroscopy," Nano Letters, vol. 10, no. 7, pp. 2511-2518, 2010.

[98] T. W. Odom, H. Gao, J. M. McMahon, J. Henzie, and G. C. Schatz, "Plasmonic superlattices: hierarchical subwavelength hole arrays," Chemical Physics Letters, vol. 483, no. 4-6, pp. 187192, 2009.

[99] W. Lee, H. Han, A. Lotnyk et al., "Individually addressable epitaxial ferroelectric nanocapacitor arrays with near $\mathrm{Tb}_{\mathrm{inch}}{ }^{-2}$ density," Nature Nanotechnology, vol. 3, pp. 402-407, 2008.

[100] Z. Fan, H. Razavi, J.-W. Do et al., "Three-dimensional nanopillar-array photovoltaics on low-cost and flexible substrates," Nature Materials, vol. 8, no. 8, pp. 648-653, 2009.

[101] S. Moghaddam, E. Pengwang, Y.-B. Jiang et al., "An inorganicorganic proton exchange membrane for fuel cells with a controlled nanoscale pore structure," Nature Nanotechnology, vol. 5, no. 3, pp. 230-236, 2010.

[102] D. Huh, B. D. Matthews, A. Mammoto, M. Montoya-Zavala, H. Yuan Hsin, and D. E. Ingber, "Reconstituting organ-level lung functions on a chip," Science, vol. 328, no. 5986, pp. 1662-1668, 2010.

[103] K.-J. Jang and K.-Y. Suh, "A multi-layer microfluidic device for efficient culture and analysis of renal tubular cells," Lab on a Chip, vol. 10, no. 1, pp. 36-42, 2010.

[104] J. B. Leen, P. Hansen, Y.-T. Cheng, and L. Hesselink, "Improved focused ion beam fabrication of near-field apertures using a silicon nitride membrane," Optics Letters, vol. 33, no. 23, pp. 2827-2829, 2008.

[105] X.-M. Yan, A. M. Contreras, M. M. Koebel, J. A. Liddle, and G. A. Somorjai, "Parallel fabrication of sub-50-nm uniformly sized nanoparticles by deposition through a patterned silicon nitride nanostencil," Nano Letters, vol. 5, no. 6, pp. 1129-1134, 2005.
[106] H. D. Tong, H. V. Jansen, V. J. Gadgil et al., "Silicon nitride nanosieve membrane," Nano Letters, vol. 4, no. 2, pp. 283-287, 2004.

[107] H. Cho, J. Kim, H. Park et al., "Replication of flexible polymer membranes with geometry-controllable nano-apertures via a hierarchical mould-based dewetting," Nature Communications, vol. 5, article 3137, 2014.

[108] Y. Yin, Y. Lu, B. Gates, and Y. Xia, “Template-assisted selfassembly: a practical route to complex aggregates of monodispersed colloids with well-defined sizes, shapes, and structures," Journal of the American Chemical Society, vol. 123, no. 36, pp. 8718-8729, 2001.

[109] Y. Xia, Y. Yin, Y. Lu, and J. McLellan, “Template-assisted selfassembly of spherical colloids into complex and controllable structures," Advanced Functional Materials, vol. 13, no. 12, pp. 907-918, 2003.

[110] P. Kim, S. E. Lee, H. S. Jung, H. Y. Lee, T. Kawai, and K. Y. Suh, "Soft lithographic patterning of supported lipid bilayers onto a surface and inside microfluidic channels," Lab on a Chip, vol. 6, no. 1, pp. 54-59, 2006.

[111] F. L. Yap and Y. Zhang, "Assembly of polystyrene microspheres and its application in cell micropatterning," Biomaterials, vol. 28, no. 14, pp. 2328-2338, 2007.

[112] S. J. Yeo, H. Kang, Y. H. Kim, S. Han, and P. J. Yoo, "Layerby-layer assembly of polyelectrolyte multilayers in threedimensional inverse opal structured templates," ACS Applied Materials and Interfaces, vol. 4, no. 4, pp. 2107-2115, 2012.

[113] G. H. Fredrickson and F. S. Bates, "Dynamics of block copolymers: theory and experiment," Annual Review of Materials Science, vol. 26, no. 1, pp. 501-550, 1996.

[114] F. S. Bates and G. H. Fredrickson, "Block copolymers-designer soft materials," Physics Today, vol. 52, no. 2, pp. 32-38, 1999.

[115] S. O. Kim, H. H. Solak, M. P. Stoykovich, N. J. Ferrier, J. J. De Pablo, and P. F. Nealey, "Epitaxial self-assembly of block copolymers on lithographically defined nanopatterned substrates," Nature, vol. 424, no. 6947, pp. 411-414, 2003.

[116] R. Ruiz, H. Kang, F. A. Detcheverry et al., "Density multiplication and improved lithography by directed block copolymer assembly," Science, vol. 321, no. 5891, pp. 936-939, 2008.

[117] J. Y. Cheng, C. T. Rettner, D. P. Sanders, H.-C. Kim, and W. D. Hinsberg, "Dense self-assembly on sparse chemical patterns: rectifying and multiplying lithographic patterns using block copolymers," Advanced Materials, vol. 20, no. 16, pp. 3155-3158, 2008.

[118] R. A. Segalman, H. Yokoyama, and E. J. Kramer, "Graphoepitaxy of spherical domain block copolymer films," Advanced Materials, vol. 13, no. 15, pp. 1152-1155, 2001.

[119] I. Bita, J. K. W. Yang, S. J. Yeon, C. A. Ross, E. L. Thomas, and K. K. Berggren, "Graphoepitaxy of self-assembled block copolymers on two-dimensional periodic patterned templates," Science, vol. 321, no. 5891, pp. 939-943, 2008.

[120] S. Park, D. H. Lee, J. Xu et al., "Macroscopic 10-terabit-persquare-inch arrays from block copolymers with lateral order," Science, vol. 323, no. 5917, pp. 1030-1033, 2009.

[121] J. K. W. Yang, Y. S. Jung, J.-B. Chang et al., "Complex selfassembled patterns using sparse commensurate templates with locally varying motifs," Nature Nanotechnology, vol. 5, no. 4, pp. 256-260, 2010.

[122] S.-M. Park, X. Liang, B. D. Harteneck et al., "Sub-10 nm nanofabrication via nanoimprint directed self-assembly of block copolymers," ACS Nano, vol. 5, no. 11, pp. 8523-8531, 2011. 
[123] J. W. Jeong, W. I. Park, L.-M. Do et al., "Nanotransfer printing with sub-10 $\mathrm{nm}$ resolution realized using directed selfassembly," Advanced Materials, vol. 24, no. 26, pp. 3526-3531, 2012.

[124] M. S. Onses, C. Song, L. Williamson et al., "Hierarchical patterns of three-dimensional block-copolymer films formed by electrohydrodynamic jet printing and self-assembly," Nature Nanotechnology, vol. 8, no. 9, pp. 667-675, 2013.

[125] J.-U. Park, M. Hardy, S. J. Kang et al., "High-resolution electrohydrodynamic jet printing," Nature Materials, vol. 6, no. 10, pp. 782-789, 2007.

[126] L. Ressier, E. Palleau, and S. Behar, "Electrical nano-imprint lithography," Nanotechnology, vol. 23, no. 25, Article ID 255302, 2012.

[127] H. O. Jacobs and G. M. Whitesides, "Submicrometer patterning of charge in thin-film electrets," Science, vol. 291, no. 5509, pp. 1763-1766, 2001.

[128] C. R. Barry, J. Gu, and H. O. Jacobs, "Charging process and Coulomb-force-directed printing of nanoparticles with sub100-nm lateral resolution," Nano Letters, vol. 5, no. 10, pp. 20782084, 2005.

[129] R. Diaz, E. Palleau, D. Poirot, N. M. Sangeetha, and L. Ressier, "High-throughput fabrication of anti-counterfeiting colloidbased photoluminescent microtags using electrical nanoimprint lithography," Nanotechnology, vol. 25, no. 34, Article ID 345302, 2014.

[130] H. Schulz, H.-C. Scheer, T. Hoffmann et al., "New polymer materials for nanoimprinting," Journal of Vacuum Science and Technology B, vol. 18, no. 4, pp. 1861-1865, 2000.

[131] L. Ressier and V. Le Nader, "Electrostatic nanopatterning of PMMA by AFM charge writing for directed nano-assembly," Nanotechnology, vol. 19, no. 13, Article ID 135301, 2008.

[132] N. Knorr, S. Rosselli, and G. Nelles, "Surface-potential decay of biased-probe contact-charged amorphous polymer films," Journal of Applied Physics, vol. 107, Article ID 054106, 2010.

[133] J. Nilsson, A. H. C. Neto, F. Guinea, and N. M. R. Peres, "Electronic properties of graphene multilayers," Physical Review Letters, vol. 97, no. 26, Article ID 266801, 2006.

[134] S. Stankovich, D. A. Dikin, G. H. B. Dommett et al., "Graphenebased composite materials," Nature, vol. 442, no. 7100, pp. 282286, 2006.

[135] A. H. C. Neto, N. M. R. Peres, K. S. Novoselov, and A. K. Geim, "The electronic properties of graphene," Reviews of Modern Physics, vol. 81, pp. 109-162, 2009.

[136] J.-A. Yan, L. Xian, and M. Y. Chou, "Structural and electronic properties of oxidized graphene," Physical Review Letters, vol. 103, no. 8, Article ID 086802, 2009.

[137] D. Choi, C. Kuru, Y. Kim et al., "Uniformly nanopatterned graphene field-effect transistors with enhanced properties," Nanoscale Research Letters, vol. 10, article 289, 2015.

[138] M. Y. Han, B. Özyilmaz, Y. Zhang, and P. Kim, "Energy bandgap engineering of graphene nanoribbons," Physical Review Letters, vol. 98, no. 20, Article ID 206805, 2007.

[139] X. Li, X. Wang, L. Zhang, S. Lee, and H. Dai, "Chemically derived, ultrasmooth graphene nanoribbon semiconductors," Science, vol. 319, no. 5867, pp. 1229-1232, 2008.

[140] X. Liang, Y.-S. Jung, S. Wu et al., "Formation of bandgap and subbands in graphene nanomeshes with sub-10 nm ribbon width fabricated via nanoimprint lithography," Nano Letters, vol. 10, no. 7, pp. 2454-2460, 2010.
[141] J. Bai, X. Zhong, S. Jiang, Y. Huang, and X. Duan, "Graphene nanomesh," Nature Nanotechnology, vol. 5, no. 3, pp. 190-194, 2010.

[142] O. Akhavan, "Graphene nanomesh by $\mathrm{ZnO}$ nanorod photocatalysts," ACS Nano, vol. 4, no. 7, pp. 4174-4180, 2010.

[143] H.-W. Li and W. T. S. Huck, "Ordered block-copolymer assembly using nanoimprint lithography," Nano Letters, vol. 4, no. 9, pp. 1633-1636, 2004.

[144] E. L. Yang, C. C. Liu, C. Y. P. Yang, C. A. Steinhaus, P. F. Nealey, and J. L. Skinner, "Nanofabrication of surface-enhanced Raman scattering device by an integrated block-copolymer and nanoimprint lithography method," Journal of Vacuum Science and Technology B, vol. 28, Article ID C6M93, 2010.

[145] X. Liang and S. Wi, "Transport characteristics of multichannel transistors made from densely aligned sub-10 $\mathrm{nm}$ half-pitch graphene nanoribbons," ACS Nano, vol. 6, no. 11, pp. 9700-9710, 2012.

[146] Z. Pan, N. Liu, L. Fu, and Z. Liu, "Wrinkle engineering: a new approach to massive graphene nanoribbon arrays," Journal of the American Chemical Society, vol. 133, no. 44, pp. 17578-17581, 2011.

[147] R. C. Schroden, M. Al-daous, and A. Stein, "Self-modification of spontaneous emission by inverse opal silica photonic crystals," Chemistry of Materials, vol. 13, no. 9, pp. 2945-2950, 2001.

[148] T. Cassagneau and F. Caruso, "Inverse opals for optical affinity biosensing," Advanced Materials, vol. 14, no. 22, pp. 1629-1633, 2002.

[149] J. S. Sakamoto and B. Dunn, "Hierarchical battery electrodes based on inverted opal structures," Journal of Materials Chemistry, vol. 12, no. 10, pp. 2859-2861, 2002.

[150] E. S. Kwak, W. Lee, N.-C. Park, J. Kim, and H. Lee, “Compact inverse-opal electrode using non-aggregated $\mathrm{TiO}_{2}$ nanoparticles for dye-sensitized solar cells," Advanced Functional Materials, vol. 19, no. 7, pp. 1093-1099, 2009.

[151] J.-H. Kim, S. H. Kang, K. Zhu, J. Y. Kim, N. R. Neale, and A. J. Frank, "Ni-NiO core-shell inverse opal electrodes for supercapacitors," Chemical Communications, vol. 47, no. 18, pp. 5214-5216, 2011.

[152] O. H. Kim, Y. H. Cho, S. H. Kang et al., "Ordered macroporous platinum electrode and enhanced mass transfer in fuel cells using inverse opal structure," Nature Communications, vol. 4, pp. 1-9, 2013.

[153] Y. H. Kim, H. Kang, S. Park et al., "Multiscale porous interconnected Nanocolander network with tunable transport properties," Advanced Materials, vol. 26, no. 47, pp. 7998-8003, 2014.

[154] K.-V. Peinemann, V. Abetz, and P. F. W. Simon, "Asymmetric superstructure formed in a block copolymer via phase separation," Nature Materials, vol. 6, no. 12, pp. 992-996, 2007.

[155] S. Y. Yang, J. Park, J. Yoon, M. Ree, S. K. Jang, and J. K. Kim, "Virus filtration membranes prepared from nanoporous block copolymers with good dimensional stability under high pressures and excellent solvent resistance," Advanced Functional Materials, vol. 18, no. 9, pp. 1371-1377, 2008.

[156] P. Zavala-Rivera, K. Channon, V. Nguyen et al., "Collective osmotic shock in ordered materials," Nature Materials, vol. 11, no. 1, pp. 53-57, 2012. 

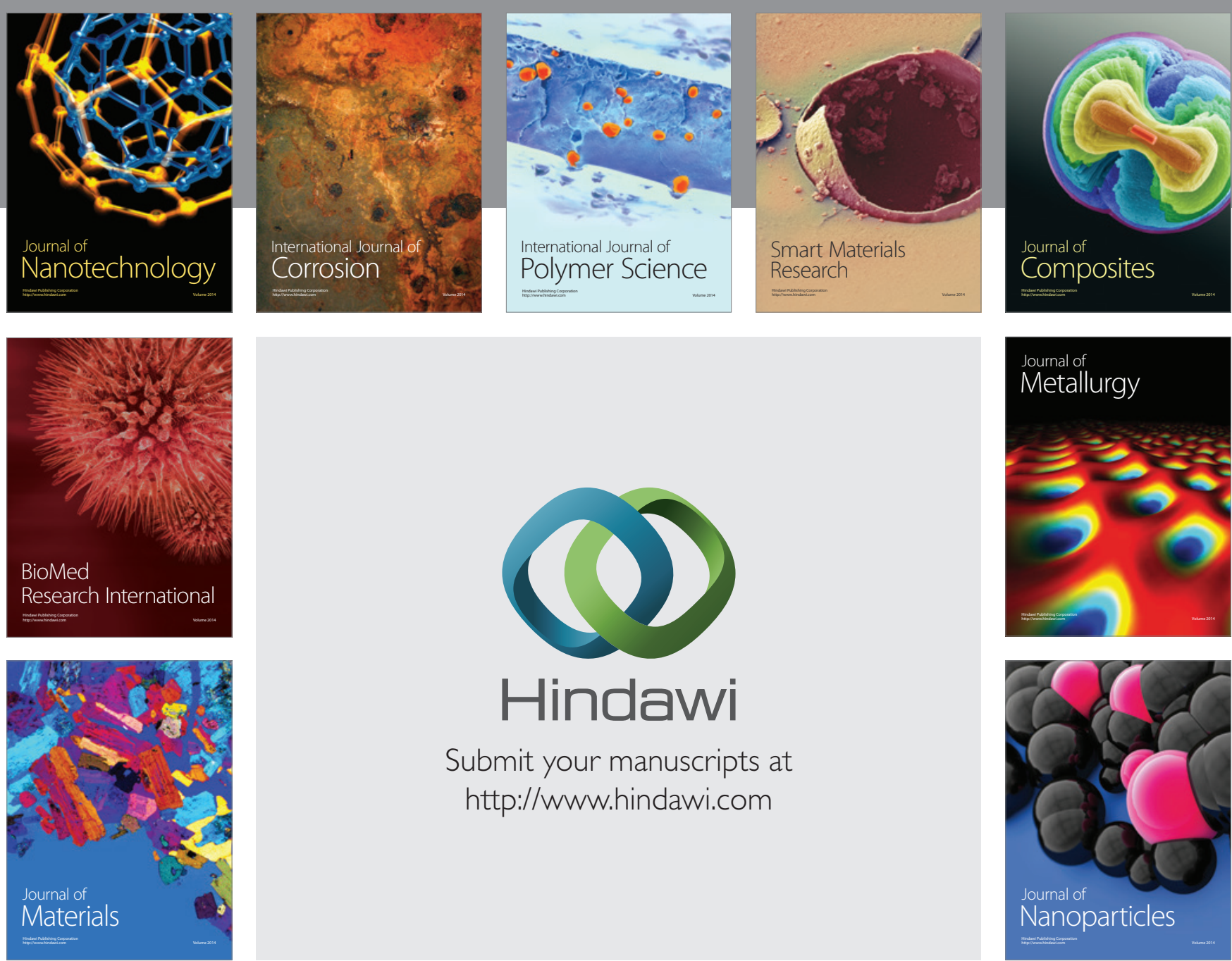

\section{Hindawi}

Submit your manuscripts at

http://www.hindawi.com

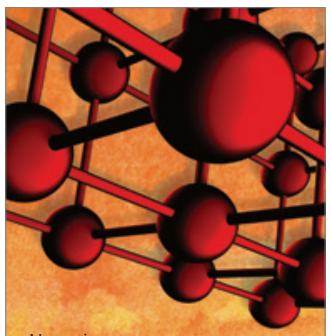

Materials Science and Engineering
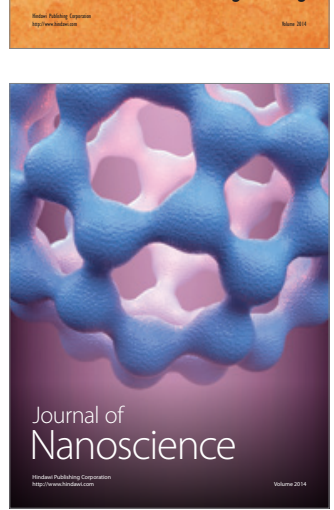
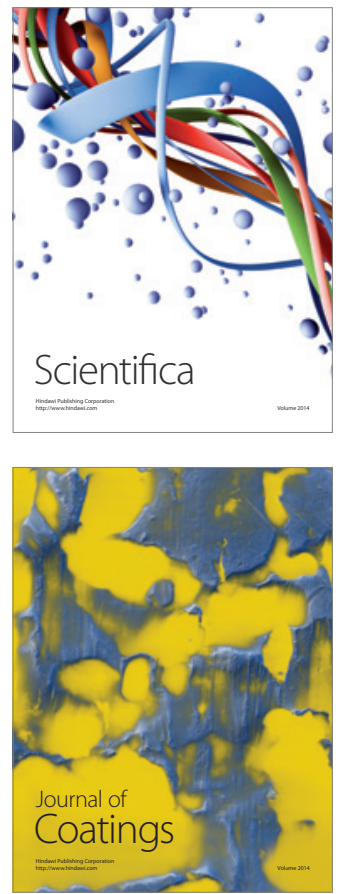
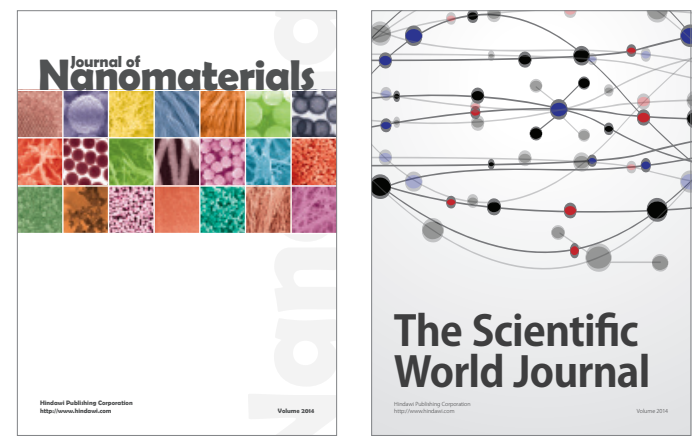

The Scientific World Journal
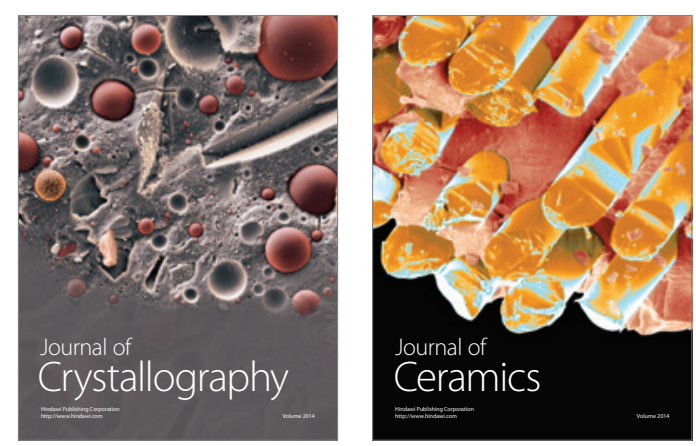
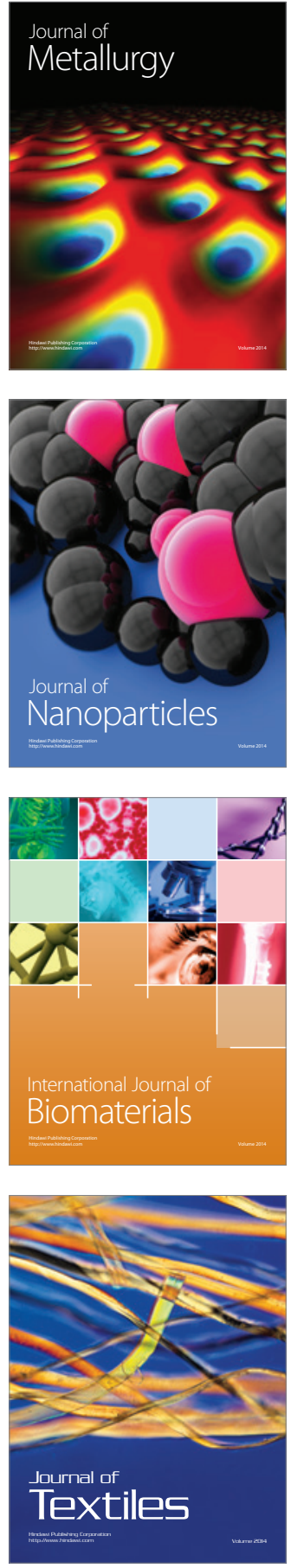